Louisiana State University

LSU Digital Commons

1973

\title{
Equilibrium States of a Dimer Model With Angular Forces.
}

\author{
Benjamin C. Freasier \\ Louisiana State University and Agricultural \& Mechanical College
}

Follow this and additional works at: https://digitalcommons.Isu.edu/gradschool_disstheses

\section{Recommended Citation}

Freasier, Benjamin C., "Equilibrium States of a Dimer Model With Angular Forces." (1973). LSU Historical Dissertations and Theses. 2460.

https://digitalcommons.Isu.edu/gradschool_disstheses/2460

This Dissertation is brought to you for free and open access by the Graduate School at LSU Digital Commons. It has been accepted for inclusion in LSU Historical Dissertations and Theses by an authorized administrator of LSU Digital Commons. For more information, please contact gradetd@lsu.edu. 


\section{INFORMATION TO USERS}

This material was produced from a microfilm copy of the original document. While the most advanced technological means to photograph and reproduce this document have been used, the quality is heavily dependent upon the quality of the original submitted.

The following explanation of techniques is provided to help you understand markings or patterns which may appear on this reproduction.

1. The sign or "target" for pages apparently lacking from the document photographed is "Missing Page(s)". If it was possible to obtain the missing page(s) or section, they are spliced into the film along with adjacent pages. This may have necessitated cutting thru an image and duplicating adjacent pages to insure you complete continuity.

2. When an image on the film is obliterated with a large round black mark, it is an indication that the photographer suspected that the copy may have moved during exposure and thus cause a blurred image. You will find a good image of the page in the adjacent frame.

3. When a map, drawing or chart, etc., was part of the material being photographed the photographer followed a definite method in "sectioning" the material. It is customary to begin photoing at the upper left hand corner of a large sheet and to continue photoing from left to right in equal sections with a small overlap. If necessary, sectioning is continued again - beginning below the first row and continuing on until complete.

4. The majority of users indicate that the textual content is of greatest value, however, a somewhat higher quality reproduction could be made from "photographs" if essential to the understanding of the dissertation. Silver prints of "photographs" may be ordered at additional charge by writing the Order Department, giving the catalog number, title, author and specific pages you wish reproduced.

5. PLEASE NOTE: Some pages may have indistinct print. Filmed as received. 
FREASIER, Benjamin C., 1945-

EQUTLIBRTUM STATES OF A DIMER MODEL WITH

ANGULAR FORCES.

The Louisiana State University and Agricultural and Mechanical College, Ph.D., 1973

Chemistry, physical

University Microfilms, A XEROX Company ، Ann Ärbor, Michigan 
EQUILIBRIUM STATES OF A DIMER MODEL WITH ANGULAR FORCES

\section{A Dissertation}

Submitted to the Graduate Faculty of the

Louisiana State University and

Agricultural and Mechanical College

in partial fulfillment of the

requirements for the degree of

Doctor of Philosophy

in

The Department of Physics

by

Benjamin C. Freasier

B.S., Louisiana State University, 1967

August 1973 


\section{ACKNOWLEDGEMENT}

I would like to acknowledge my debt to Professor Runnels for his guidance and patience. Without his insight this study would certainly not have been possible.

I would like to acknowledge my wife's patience and understanding during this pertod of our lives.

I am also deeply obligated to my parents who took a strong interest In my education, often with sacrifice to themselves.

Acknowledgement is also due the Dr. Charles E. Coates Memorlal Fund for assistance in publishing this dissertation.

Acknowledgement is also due to the National Sclence Foundation whose grant to this institution made this work possible. 
TABLE OF CONTENTS

I. INTRODUCTION

II. ANALYTIC REGION FROM YANG-LEE-RUELLE THEOREM 6

III. UNIQUENESS REGION IN THE $\beta-\mu$ HALF-PLANE BY DOBRUSHIN'S THEOREM 15

IV. NON-UNIQUENESS REGION IN THE $\beta-\mu$ HALF-PIANE 21

V. PERTURBATION CALCULATION--LOW TEMPERATURE LIMIT $\quad 29$

VI. SOME SPECIFIC EXAMPLES 32

VII. CONCLUSION 34 
1. Nearest neighbor pair interactions.

2. Close-packed structures.

3. The auxiliary lattice.

4. The YLR analytic region for $a=4 b$.

5. The ten sites that interact with the origin in the auxiliary lattice.

6. A configuration with nematic boundary conditions.

7. A configuration with smectic boundary conditions.

8. The configuration obtained from Figure 6 by $\mathrm{T}$.

9. The configuration obtained from Figure 7 by $T$.

10. State diagram for $a=4 b<0$.

11. State diagram for $a=b<0$.

12. State diagram for $a=3 b>0$. 


\section{ABSTRACT}

Several complementary techniques are applied to the study of the orientational transition in a restricted lattice model of rigid linear dimers with finite interactions between contiguous molecules, on the square lattice. The restriction has the effect of forcing the ordered phase to resemble either a smectic or a nematic liquid crystal. It is shown that the symmetry of the equilibrium state is broken for some interactions and that the equilibrium state is unique for others. Thermodynamic analyticity is established for high temperatures. 
I. INTRODUCTION.

A. The Genera1 Problem. Considerable analytical and numerical work has been done on the study of dense fluids and their phase transitions. With the increased interest in the behavior of 1iquid crystalline systems, a similar model applicable to such systems has emerged--rigid straight polymeric molecules that are constrained to fit on some lattice. These lattice gas systems can be studied from a number of viewpoints, but the principal objective of this work is to derive rigorous results that shed some light on the nature of the presumed phase transition for a specific example of this type of mode1--the restricted dimer mode1.

B. Restricted Dimer Mode1. The underlying lattice for this mode1 is the square lattice. On this lattice are placed dimers whose width is the edge of the unit cell of the square lattice. The dimer may be of horizontal or vertical orientation, but no two molecules may overlap. The model is further restricted for mathematical simplicity: the left end of a horizontal dimer and the bottom of a vertical dimer must reside on a lattice site whose coordimates $(x, y)$ satisfy the condition $\mathrm{x}=\mathrm{y}(\bmod 2)$. Such a lattice site is denoted a reference site. These reference sites make up the restricted square lattice. A reference site is not necessarily occupied by a dimer.

The dimers may interact by way of a nearest-neighbor pair potential. For two adfacent parallel dimers, the pair potential is a; for two perpendicular dimers, the pair potential is $b$ (Figure 1 ). It is assumed that not both $a$ and $b$ are zero. The other nearest neighbor interaction, c, for end to end dimers is also of interest; however, a fundamental transformation introduced in Section IV is invalidated for 
non-zero c. So, $c$ is set equal to zero. A11 other pair and higher order many-body potentials are set equal to zero.

Two interesting types of close-packed ordered structures for the restricted dimer model are shown in Figure 2. These structures have the appearance of the phase structures of nematic and smectic 1iquid crystals (intermediate phases that some organic solids go through in melting). Those names will be used to denote these two close-packed ordered structures. The interaction energy per dimer for the closepacked nematic (smectic) structure is $2 a(a+2 b)$. The nematic (smectic) structure is energetically favored if $a<2 b(a>2 b)$.

C. Genera1 Outline of Methods Used in Study.

1. Yang-Lee-Rue1le Theorem. It is we11 known that dimers on the unrestricted lattice do not have a phase transition if they do not interact except for the hard core exclusion. ( See $1,2,3,4$. ) For the sake of completeness, this will be shown by the Yang-Lee-Ruelle Theorem (5) for the restricted dimer problem. The Yang-Lee-Ruelle (YLR). Theorem locates regions in the complex activity plane (activity= $\exp (\beta \mu), \beta=1 / k T, \mu=c h e m i c a 1$ potential, $T=$ temperature, and k=Boltzman's constant) where the Grand Canonical Partition Function for the lattice gas, $z$, does not have any zeros. In such regions the thermodynamic functions must be analytic with respect to $z$, since they depend upon $\log (z)$. It wi11 be shown that such a region can be found that includes the entire real positive axis. This implies that the thermodynamic functions are analytic; hence, this region must be of one phase. This Idea follows from the conventional association of nonanalyticity of a thermodynamic function with a phase change. In addition, if $a$ and $b$ are not both zero, this theorem will be used to find 
a connected region in the $\beta-\mu$ half-plane where $z$ is analytic; hence, the system in this region must be of one phase.

2. Dobrushin's Uniqueness Theorem. Dobrushin (6) has introduced the idea of a random field and its distribution applied to square lattice gas systems. This random field is the occupation numbers of a11 the lattice sites. In addition, this fleld and its distribution is called "Gibbsian" if the probability of a configuration $\alpha$ is given by $\mathrm{q}_{\mathrm{V}}=\exp [-\beta \mathrm{U}(\alpha)] / \mathrm{Z}$, where $\mathrm{U}(\alpha)$ is the free energy of the configuration $\alpha$ in $V$ for all finite $V$ in a square lattice. Dobrushin has claimed that when the Gibbsian distribution for specified $\beta, \mu$, and potential is unique that there is no separation of phases $(6,7)$. Essentially, uniqueness of the Gibbsian distribution implies that the occupation number of one lattice site must not unduly influence the probability of a site being occupied. If $T$ is large enough, the probability of a site being occupled should be close to the corresponding probability for an ideal gas for which the molecules are relatively independent. As $\mu \rightarrow-\infty$, the probability of any site being occupied is close to zero for any condition, so the occupation of one site does not greatly affect the probability of another site being occupied. Dobrushin ( 7 ) has proved a theorem (which will be used in Section III) that systematizes the conditions under which local perturbations in a configuration do not greatly influence the Gibbsian distribution. 3. Contour Method. While it can be easily shown that this model does not have a phase transition for $a=b=0$, it is not at all obvious for arbitrary values of $a$ and $b$ that a phase transition will occur for low enough $T$. As a matter of fact, the relative roles of attractive and repulsive forces in mesomorphic transitions has never been settled. 
It is natural to presume that the analytic region in the $\beta-\mu$ halfplane deduced by the YLR Theorem and the random field uniqueness region In the $\beta-\mu$ half-plane deduced by Dobrushin's Uniqueness Theorem represent the same phase, specifically, the randomly oriented phase. If Indeed, a phase transition occurs, it is to be expected that an orientational transition will take place to the nematic or smectic closepacked structure. The question is whether or not the system can sustain long range order through short range interactions. This type of question has been answered for a number of models using the contour method $(7,8,9,10)$.

The basic line of reasoning has several steps. Close-packed molecules of nematic (smectic) structure are placed outside the volume V. Contours are drawn around groups of dimers of misaligned structure. The probability of such a contour is derived. It is noted that the probability that a reference site is occupled by a dimer of the "wrong" structure is bounded above by the sum of the probabilities of occurence of a11 contours that can arise from "wrong" dimers being in the vicinity of the reference site. It is then shown that this probability is less than $1 / 2$ for low enough temperature. The boundary conditions are then changed slightly so that if a dimer at a reference site was "wrong" it is now "right" for the altered boundary conditions. (The boundary structure must still be nematic (smectic).) The same arguments are repeated. If a11 this can be shown to independent of the size of $\mathrm{V}$, then the system can support long range order through short range interactions. This is true since the alignment of a particular dimer depends on the alignment of the boundary dimers which may be quite distant from the reference site in question. 
4. Perturbation Method. If it is assumed at low temperatures that there coexists a random phase and a close-packed phase, then a zeroth order perturbation calculation can be made by equating the chemical potentials and the pressures of each phase. It must be stressed that such a coexistence is an assumption. It does, however, provide a useful, if not completely rigorous, asymptote for the presumed phase coexistence interface. The calculation is done in Section V. 
II. ANALYTIC REGION FROM YANG-LEE-RUELLE THEOREM.

A. Yang-Lee-Rue1le Theorem. For a lattice gas system, Z (Grand Partition Function) is given by

$$
z=\sum_{\alpha} \exp \left[\beta \sum_{i=1}^{V} \mu x_{1}\right] \exp \left[-\beta U\left(x_{1}, \ldots, x_{v}\right]\right.
$$

where $U$ is the potential energy of the gas, $x_{1}$ is the occupation number of the $1^{\text {th }}$ site ( 0 or 1 ), and $\alpha$ labels configurations. If a different activity is associated with each site $\left(z_{1}=\right.$ site activity $=$ $\left.\exp \left[\beta \mu x_{1}\right]\right)$, then

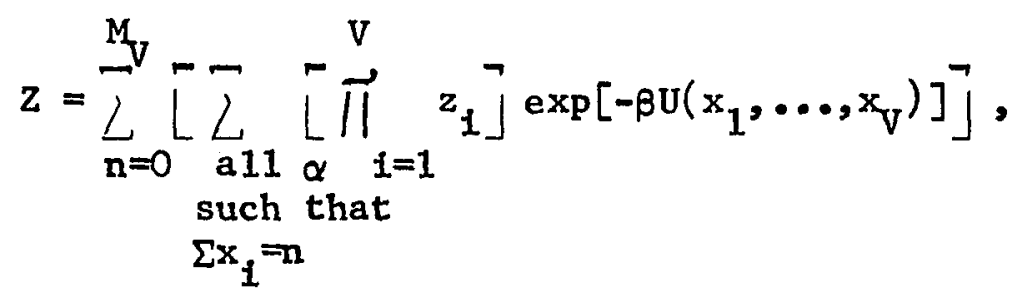

where $\mathrm{M}_{\mathrm{V}}$ is the maximum number of particles that can be packed into $\mathrm{V}$. If, as is customarily done, all the site activities are set equal to $z$, then

$$
z=\sum_{n=0}^{M} z_{n} z^{n},
$$

where $z_{n}$ is the canonical partition function for $n$ particles. Another way of writing $z$ is

$$
z=z(v, z)=\overrightarrow{\|}\left(1-z / w_{v}\right)
$$

where $w_{\nu}$ are the $\nu=1, \ldots, M_{V}$ solutions to the equation $z(v, z)=0$. If the limits exits (for the bulk thermodynamic 1imit), the following 
relations hold:

$$
\begin{aligned}
& \text { Pressure } / \mathrm{kT}=\mathrm{P} / \mathrm{kT}=\lim _{\mathrm{V} \rightarrow \infty} \frac{1}{\mathrm{~V}} \log \left(\mathrm{z}_{\mathrm{V}}\right) \\
& \text { Density }=\rho=\lim _{V \rightarrow \infty} \frac{\partial}{\partial(\log z)} \frac{1}{V} \log \left(z_{V}\right) .
\end{aligned}
$$

Gruber and Kunz (2) have shown that these limits do exist. Al1 the $Z_{n}$ are positive, so for finite $M_{V}$, no root can be a real positive number. As $V$ increases, the roots move about in the complex plane, and the ir number increases linearly with $V$. The distribution of these roots as $\mathrm{V} \rightarrow \infty$ gives the complete analytic behavior of the defined thermodynamic functions. So, if some of the roots close in on the posItive rea1 axis in the bulk thermodynamic limit, the re may be singular behavior in $P$ and $\rho$ indicating a phase transition. If $z(z) \neq 0, P$ and $\rho$ are analytic functions of $z$.

The YIR Theorem is a general theorem to find regions in the complex $z$ plane for which $z(z) \neq 0$ (for any interaction potentials between particles of a lattice gas). Let $\Omega$ denote the sites of some lattice $(|\Omega|<\infty)$. Let $\alpha$ index a finite collection of sublattices $\left(\Omega_{\alpha}\right)$. If the collection of sublattices $\left\{\Omega_{\alpha}\right\}$ contains a11 the sites of $\Omega$ and a11 the possible potential interactions of the main lattice, it is a

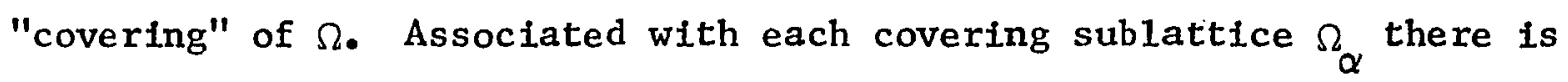
a grand canonical partition function $z_{\alpha}$ that is a function of as many site activities $\left(\left|\Omega_{\alpha}\right|\right)$ as there are sites in $\Omega_{\alpha^{*}}$ Associated with eachach $\alpha$ and each site activity $i$ in $\Omega_{\alpha}$ is a set of regions of the complex plane, denoted $M_{\alpha, i}$, with the following properties: 
1. $M_{\alpha, 1}$ is closed.
2. $0 \& M_{\alpha, 1}$.

Neither the choice of $\left\{\Omega_{\alpha}\right\}$ or $M_{\alpha, 1}(1=1, \ldots,|\Omega|)$ is unique for any given problem.

YANG-LEE-RUELLE THEOREM. If the regions $M_{\alpha, 1}$ are chosen (for each $\alpha$ ) so that the requirement $z_{1} \in \sim M M_{\alpha, 1}$ for al1 sites in $\Omega_{\alpha}$, insures that $z_{\alpha} \neq 0$, then the similar statement can be made for the entire lattice: $z_{1} \in \sim R_{1}$ for all the sites in $\Omega$ insures that $z_{\Omega} \neq 0$, where $R_{1}$ is the region of the complex plane given symbolically by the "set product" $R_{1}=-\pi_{\alpha}\left(-M_{\alpha, i}\right)$, the product being over all values of $\alpha$ such that $\Omega_{\alpha}$ contalns a site activity of site $i$. If the intersection over all values of $i=1, \ldots,|\Omega|, s=\bigcap_{1} \sim R_{i}$ is not empty, then $z(z) \neq 0$ in $s$. The symbols $\sim \mathrm{X}$ stands for the complement of the set $\mathrm{X}$. B. Restricted Dimer Model without Angular Forces. The 1attice gas as defined thus far has an interaction ambiguity associated with each site. (The dimer can be oriented two ways.) This problem can be circumvented by using an auxiliary lattice which is formed by placing lattice sites at the midpoints of lines connecting nearest neighbors of the restricted square lattice (4). An orientation is automatica11y assoctated with each auxiliary site. An $\mathrm{x}$ will be used to denote a vertical dimer site, and an $o$ will be used to denote a horizontal dimer site. A heavy line will be used to represent a forbidden configuration between two sites. A light line will be used to represent an 'a' interaction. A broken line will be used to represent $a$ ' $b$ ' interaction. A portion of the auxiliary lattice is shown in Figure 3 . Note that if $a=$ $b=0$, the interaction is one-dimensional; hence, there can be no phase transition since this model has finite range interactions (12). 
For $a=b=0$, the lattice $\Omega$ can be covered by two-point lattices

and their interactions. Every lattice site is covered twice. So, each $R_{1}$ Involves the set product of two regions in the complex plane. The partition function for $\Omega_{\alpha}$ can be written down immediately. (They are al1 the same except for dummy variables.)

$$
z_{\alpha}=1+z_{1}+z_{2}
$$

This has been examined previously (4), but a completely rigorous proof had not been offered. Let $M=M_{\alpha, 1}=M_{\alpha, 2}=\{z \mid \operatorname{Re} z \leq-1 / 2\}$. So,

$$
R_{1}=\left\{-z_{1} z_{2} \mid z_{1} \in M, z_{2} \in M\right\}
$$

To find the boundary of $R_{i}$, the following lemma is needed. LEMMA. Given that $z_{i} \in M_{1}$ and $z_{j} \in M_{j}, O \notin M_{i}, O \notin M_{j}$, then the boundary of the set product of $M_{1} M_{j}=R$ must come from the product of boundary points from $\mathrm{M}_{i}$ and $\mathrm{M}_{\mathrm{j}}$.

Proof: To say that there is a point $\mathrm{p}$ interior to $\mathrm{R}$ is to say that there exists a neighborhood $\epsilon>0$ such that all points $q$ in this neighborhood belong to $R$, or

$$
|p-q|<\epsilon, \quad p \in R, q \in R \text {. }
$$

Let $p=z_{1} z_{j}$, with at least one $z$ (say $z_{j}$ ) an interior point. Ca11 $z_{1}=l$. (It is here that $z_{i}$ could be replaced by a product of $z^{\prime} s$ from various regions without affecting the argument. This would allow the extension of this lemma to set products of more than two sets.)

So, $\left|\ell z_{j}-q\right|<\epsilon$ is what is wanted.

Pick $q=\ell\left[\left(x_{j}+\delta_{1}\right)+i\left(y_{j}+\delta_{2}\right)\right]$.

Since $z_{j}$ is an interior point of $M_{j}$, it must be bounded away from the boundary line of the set. So, pick $\delta_{1}$ and $\delta_{2}$ such that $\left(x_{j}+\delta_{1}\right)+\left(y_{j}+\delta_{2}\right)$ is still interior to $\mathrm{M}_{j}$. Call the smaller of the maximum $\left|\delta_{1}\right|,\left|\delta_{2}\right|$ such that the preceding relationship is satisfied $\Delta$. Then any point of 
the form $\left(x_{j}+\delta_{1}\right)+1\left(y+\delta_{2}\right),\left|\delta_{1}\right|<\Delta,\left|\delta_{2}\right|<\Delta$ is interior to $M_{j}$. It is necessary to be sure that all points $q$ are covered in the neighborhood $\epsilon$ or $l\left(x_{j}+i y_{j}\right)-l\left[\left(x_{j}+\delta_{1}\right)+i\left(y_{j}+\delta_{2}\right)\right]=k_{1}+i k_{2}$, where $\left|\kappa_{1}\right|^{2}+\left|\kappa_{2}\right|^{\prime 2}<\varepsilon^{2}$. These equations can be easily solved since $\ell \neq 0$. The fundamental relations are

$$
\begin{aligned}
& \operatorname{Re}(\ell) \delta_{1}+\operatorname{Im}(\ell) \delta_{2}=\kappa_{1} \\
& \operatorname{Im}(\ell) \delta_{1}+\operatorname{Re}(\ell) \delta_{2}=\kappa_{2} .
\end{aligned}
$$

So,

$$
\begin{aligned}
& \delta_{1}=\frac{\kappa_{1} \operatorname{Re}(\ell)-\kappa_{2} \operatorname{Im}(\ell)}{|\ell|^{2}} \\
& \delta_{2}=\frac{\kappa_{2} \operatorname{Re}(\ell)-\kappa_{1} \operatorname{Im}(\ell)}{|\ell|^{2}}
\end{aligned}
$$

The inverse has been found that fills the neighborhood $\varepsilon$. Pick $\epsilon=|\ell| \Delta$. Certain1y, all points in the neighborhood can be found, the question is whether or not a11 $q$ are from points interior to $\mathrm{M}_{\mathbf{j}}$. We11,

$$
\begin{aligned}
& |p-q|<\epsilon=|\ell| \Delta, \\
& \left|\ell z_{j}-\ell\left[\left(x_{j}+\delta_{1}\right)+1\left(y_{j}+\delta_{2}\right)\right]\right|<|\ell| \Delta, \\
& \left|\delta_{1}+1 \delta_{2}\right|<\Delta .
\end{aligned}
$$

So, $\left|\delta_{1}\right|<\Delta$, and $\left|\delta_{2}\right|<\Delta$, which implies that the point must come from the interior of $\mathrm{M}_{\mathrm{j}}$. Since any set products of points coming from the interfor contain at least one multiplicand from the interior of one of the multiplicand sets, the boundary points of the set product must come from the boundary points of the sets involved. Q.E.D.

In this case, the boundary of $R_{i}=R$ (for all $i$ ) comes from the product of points of the form $-1 / 2+1 t$. So, a typical boundary point looks like $\left(t_{1} t_{2}-1 / 4\right)+i 1 / 2\left(t_{1}+t_{2}\right)$. Not a11 these points are boundary points of the set product, but the boundary must come from a product of this 
sort. The primary area of interest is how close the boundary of the set power comes to the positive real activity axis. If $x=t_{1} t_{2}-1 / 4$, for a given $x$ the object is to minimize $y^{2}$. It is apparent that

$$
\begin{aligned}
& f=y^{2}=1 / 4\left[(x+1 / 4) / t_{1}+t_{1}\right], \\
& \partial f=0=\frac{1}{2}\left\lfloor\frac{x+1 / 4}{t_{1}}+t_{1}\right\rfloor\left[\frac{x+1 / 4}{-t_{1}^{2}}+1\right\rfloor .
\end{aligned}
$$

So, $t_{1}=t_{2}$. It is easy to show that this is a minimum for $x>-1 / 4$ by noting that the second derivative is positive at $t_{1}=t_{2}$. Hence, the set product is bounded by the parabola $t^{2}-1 / 4+i t$ and $R$ is excluded from the positive real axis. Clearly, $s=\bigcap_{i} \sim R_{i}=\sim R$ so there are no zeros in $Z$ for any positive real $z$, hence, no phase transition. It might be noted that $M$ could have been chosen to be the half-plane excluded from zero by any straight line going through $x=-1 / 2$. Then analagous arguments show that $Z$ cannot have any zeros on the line perpendicular to this line. This argument is valid for any such line that does not go through the orfgin: therefore, this implies that any zeros of $\mathrm{Z}$ must be on the negative real axis as for the unrestricted model (1).

C. Dimer Model with Angular Forces. In this case there are several more interactions to consider. However, for high temperatures the $a$ and b interactions become ineffective. If a particular site in the auxiliary lattice is picked out (cal1 it $i=1$ ), then its interactions can all be covered by 10 two-point lattices and their interactions. There are four two-point lattices with a interactions $(\alpha=1,4)$, four two-point lattices with $\mathrm{b}$ interactions $(\alpha=5,8)$, and two two-point lattices with forbidden interactions $(\alpha=9,10)$. The partion functions for these covering lattices can be immediately written down (using $z_{2}$ as a dummy varlable): 


$$
\begin{aligned}
& z_{1}=z_{2}=z_{3}=z_{4}=1+z_{1}+z_{2}+z_{1} z_{2} \exp (-\beta a), \\
& z_{5}=z_{6}=z_{7}=z_{8}=1+z_{1}+z_{2}+z_{1} z_{2} \exp (-\beta b), \\
& z_{9}=z_{10}=1+z_{1}+z_{2}
\end{aligned}
$$

From Ruelle (5) it is known that

$$
\begin{aligned}
& M_{(1,2,3,4), 1}=\left\{z|| z+1 \mid \leq \exp (\beta a)[1-\exp (\beta a)]^{1 / 2}\right\}, a \leq 0 . \\
& M_{(1,2,3,4), 1}=\left\{z|| z+\exp (\beta a) \mid \leq \exp (\beta a)[1-\exp (-\beta a)]^{1 / 2}\right\}, a>0 .
\end{aligned}
$$

It would be desirable to find a circle centered on $z=-1$ which would in-



Let $\beta=\log x / a, 1<x<4 / 3$.

Then the smallest point on the negative real $z$ axis intersected by $M_{(1,2,3,4), 1}$ is $-x(1+\sqrt{1-1 / x})$. The question is whether or not there exists an $a^{\prime}$ such that the radius of a circle around -1 includes this

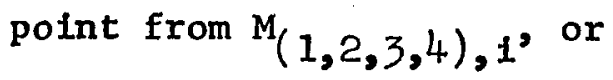

$$
\left[1-\exp \left(\beta \mathrm{a}^{\prime}\right)\right]=\mathrm{x}(1+\sqrt{1-1 / \mathrm{x}})-1 \text {. }
$$

This can be solved to yield

$$
a^{\prime}=\frac{a \log \left\{1-[x(1+\sqrt{1-1 / x})-1]^{2}\right\}}{\log x}
$$

So, Ruelle's $M$ is included in the region

$$
M=\left\{z|| z+1 \mid \leq\left(1-e^{-\beta\left|a^{\prime}\right|}\right)=A\right\} \text {, }
$$

where $a^{\prime}$ is given above for $a>0$ and equals a for $a \leq 0$. Similar remarks can be made about the $b$ interactions. From the previous discussion, it is known that sufficient regions for the forbidden interactions are

$$
M_{(9,10), 1}=\{z \mid \operatorname{Re} z \leq-1 / 2\} \text {. For the moment, the set product of } M_{1}
$$
through $M_{1}$ will be taken. Points in this set product are of the form:

$$
\pi=\left(-1+\xi_{1}\right)\left(-1+\xi_{2}\right)\left(-1+\xi_{3}\right)\left(-1+\xi_{4}\right)\left(-1+\xi_{5}\right)\left(-1+\xi_{6}\right)\left(-1+\xi_{7}\right)\left(-1+\xi_{8}\right),
$$

where $\left|\xi_{1}\right| \leq A, 1=1,4 ;\left|\xi_{1}\right| \leq B, i=5,8$.

This product can be expanded in terms linear in $\xi_{i}$ : 


$$
\begin{aligned}
\pi= & 1+\xi_{1}\left[1+1 / 2\left(\xi_{2}+\ldots+\xi_{8}\right)+1 / 3\left(\xi_{2} \xi_{3}+\ldots+\xi_{7} \xi_{8}\right)+1 / 4\left(\xi_{2} \xi_{3} \xi_{4}+\ldots+\right.\right. \\
& \left.\xi_{6} \xi_{7} \xi_{8}\right)+1 / 5\left(\xi_{2} \xi_{3} \xi_{4} \xi_{5}+\ldots+\xi_{5} \xi_{6} \xi_{7} \xi_{8}\right)+1 / 6\left(\xi_{2} \xi_{3} \xi_{4} \xi_{5} \xi_{6}+\ldots+\xi_{4} \xi_{5} \xi_{6}\right. \\
& \left.\left.\xi_{7} \xi_{8}\right)+1 / 7\left(\xi_{2} \xi_{3} \xi_{4} \xi_{5} \xi_{6} \xi_{7}+\ldots+\xi_{3} \xi_{4} \xi_{5} \xi_{6} \xi_{7} \xi_{8}\right)+1 / 8\left(\xi_{2} \xi_{3} \xi_{4} \xi_{5} \xi_{6} \xi_{7} \xi_{8}\right)\right] \\
& +\ldots
\end{aligned}
$$

where $+\ldots$ stands for similar terms linear in $\xi_{i}(i \neq 1)$ designed to make the product symmetrical. The absolute value of $\pi^{-1}$ is denoted by $Q$. Since $A<1$, and $B<1$,

$$
\begin{aligned}
& Q \leq\left[7+\frac{1}{2}\left(\begin{array}{l}
7 \\
1
\end{array}\right)+\frac{1}{3}\left(\begin{array}{l}
7 \\
2
\end{array}\right)+\frac{1}{4}\left(\begin{array}{l}
7 \\
3
\end{array}\right)+\frac{1}{5}\left(\begin{array}{l}
7 \\
4
\end{array}\right)+\frac{1}{6}\left(\begin{array}{l}
7 \\
5
\end{array}\right)+\frac{1}{7}\left(\begin{array}{l}
7 \\
6
\end{array}\right)+\frac{1}{8}\left(\begin{array}{l}
7 \\
7
\end{array}\right)\right] \\
& x\left[\left|\xi_{1}\right|+\left|\xi_{2}\right|+\left|\xi_{3}\right|+\left|\xi_{4}\right|+\left|\xi_{5}\right|+\left|\xi_{6}\right|+\left|\xi_{7}\right|+\left|\xi_{8}\right|\right] .
\end{aligned}
$$

So, $Q \leq(255 / 2)(A+B)$ for small enough $\beta$. From the last section it is known that the set product must be taken of $L^{\bullet} \pi$, which gives the desired region $R_{1}$. Elements of $\pi$ can be represented by points of the form:

$$
1+\xi,|\xi|<Q, \xi=x+i y \text {. }
$$

From the Lemma proved in Paragraph A of this section, it is known that the boundary of this set product must come from boundary points of the sets involved in the product, or points of the form:

$$
\left[t^{2}-1 / 4-i t\right][(1+x)+i y]=\left[\left(t^{2}-1 / 4\right)(1+x)+t y\right]+i\left[-t(1+x)+y\left(t^{2}-1 / 4\right)\right] \text {. }
$$

The specific problem is to determine where points of this form can be on the real positive axis. So, assume that

$$
\begin{aligned}
& -t(1+x)+y\left(t^{2}-1 / 4\right)=0, \text { or } \\
& t=\frac{(1+x) \pm \sqrt{(1+x)^{2}+y^{2}}}{2 y}, y \neq 0 .
\end{aligned}
$$

(If $y=0$, the points all fall on a parabola which never touches the positive real axis.) The positive real intercept, $z_{\text {int }}$, can then be written: 


$$
\begin{aligned}
z_{\text {int }}= & {\left[\frac{(1+x)^{2} \pm 2(1+x) \sqrt{(1+x)^{2}+y^{2}}+(1+x)^{2}+y^{2}}{4 y^{2}}-1 / 4\right](1+x) } \\
& +\frac{(1+x) \pm \sqrt{(1+x)^{2}+y^{2}}}{2}
\end{aligned}
$$

The solution with the negative sign is negative, so the positive sign case is the only one that need be considered. Hence,

$$
\begin{aligned}
& z_{\text {int }}=\exp \left(\beta \mu_{\text {int }}\right) \leq\left[(1-Q)^{2} / Q^{2}+3 / 4\right](1-Q), \text { and } \\
& \mu_{\text {int }} \leq(1 / \beta) \log \left[(1-Q)\left[(1-Q)^{2} / Q^{2}+3 / 4\right]\right] \text {, where } \\
& Q=(255 / 2)\left[\left(1-e^{-\beta\left|a^{\prime}\right|}\right)+\left(1-e^{-\beta\left|b^{\prime}\right|}\right)\right]<1 .
\end{aligned}
$$

As $\beta \rightarrow 0, Q \rightarrow 0$, and $\mu_{\text {int }} \rightarrow+\infty$. The restriction $Q<1$ puts a limit on how large $\beta$ can be as $\mu_{\text {int }}{ }^{-\infty}$. An example of the YLR analytic region with $a=4 b<0$ is shown in Figure 4. This graph is qualitatively similar for any value of $a$ and $b$. The $\beta$ limit can be calculated explicitly for $a$ or $b$ equal to zero. Assume $b=0$. Then

$$
\begin{aligned}
& (255 / 2)\left(1-e^{-\beta\left|a^{\prime}\right|}\right)^{1 / 2}<1 \text {, or } \\
& \beta<6 \times 10^{-5} /|a| \text {, after the appropriate algebra. }
\end{aligned}
$$

The conclusions that can be drawn from the entire analysis is that there is an analytic region in the $\beta-\mu$ plane for all $\mu$ for small enough $\beta$. It is not to be construed from this presentation that the best choices of M's and the most clever manipulation of the set products were made. 
III. UNIQUENESS REGION IN THE $\beta-\mu$ HALF-PIANE BY DOBRUSHIN'S THEOREM.

A. Dobrushin's Uniqueness Theorem. Dobrushin's notation will be followed as closely as possible ( 7 ). A configuration on the auxiliary lattice is a function $x$ from the lattice to the discrete space $\{0,1\}$. So, $x(t)=1$ if the site $t$ is occupied, and $x(t)=0$ if the site is empty. A different configuration is described by a different function $x(t)$. The single variable $x$ with no argument is identical to $x(0)$ which is the characteristic function for the site specified as the origin. The notation $q(x \mid x(t))$ stands for the conditional probability that the origin has the value $x$. In other words, for Gibbsian distributions

$$
q(x \mid x(t))=\frac{\exp [-\beta U(x ; x(t))]}{\sum_{x=0,1} \exp [-\beta U(x ; x(t))]},
$$

where $U(x ; x(t))$ is given by

$$
U(x ; x(t))=-\mu x+1 / 2 \sum_{i=1}^{\infty} x x_{i} U\left(t_{i}\right),
$$

where $U(t)$ is the potential interaction. The Dobrushin Uniqueness Theorem can be stated as

$$
\Delta<1 / 2 \sum_{s \neq 0}(\sup )_{s} \sum_{x=0,1}|q(x \mid x(t))-q(x \mid \tilde{x}(t))|<1,
$$

where (sup) means the supremum taken over all pairs of characteristic functions $x(t)$ and $\widetilde{x}(t)$ which agree everywhere except possibly at $t=s$. B. Application to Restricted Dimer Problem. The summation over $s$ in $\Delta$ is limited to ten sites since $U(t)$ is zero elsewhere. A labeled portion of the auxiliary lattice is shown in Figure 5. Define

$$
\begin{aligned}
\Delta_{S} & \left.=1 / 2 \sum_{x=0,1} \mid q(x \mid x(t))-q(x \mid \widetilde{x})\right) \mid \\
& =\left|\left\{1+\exp \left[\beta \mu-\sum_{t \neq 0} U(t) x(t)\right]\right\}^{-1}-\left\{1+\exp \left[\beta \mu-\sum_{t \neq 0} U(t) \widetilde{x}(t)\right]\right\}^{-1}\right| .
\end{aligned}
$$


Consider $s=1(2,3,4)$. Clearly, $\Delta_{s}$ vanishes if $x(s)=\tilde{x}(s)$, or if $x(9)$ or $x(10)=1$. Otherwise,

$$
\Delta_{\mathrm{S}}^{\mathrm{C}}=\frac{\mathrm{Cz}|\mathrm{B}-1|}{(1+\mathrm{Cz})(1+\mathrm{CzB})},
$$

where $A=e^{-a \beta}, B=e^{-b \beta}, C=e^{-\beta \Sigma} U(t) x(t), z=e^{\beta \mu}$. It is assumed that $x(s)=0$ and $\tilde{x}(s)=1$. Similarly, for $s=5(6,7,8)$,

$$
\Delta_{\mathrm{S}}^{\mathrm{C}}=\frac{\mathrm{Cz}|\mathrm{A}-1|}{(1+\mathrm{Cz})(1+\mathrm{CzA})} \text {. }
$$

For $s=9, \Delta_{9}$ vanishes unless $x(9) \neq \tilde{x}(9)=1$, provided $x(10)=0$. So,

$$
\Delta_{\mathrm{S}}^{\mathrm{C}}=\mathrm{Cz} /(1+\mathrm{Cz}) \text {. }
$$

A similar answer is obtained for $s=10$. The objective is to determine the supremum $\Delta_{S}$. This will be done by maximizing $\Delta_{s}$ through variations in $C$. What is wanted is $\Delta_{s}^{C} \leq \Delta_{s} C_{2}$. This is true for $s=9$ if

$$
\frac{C_{1} z}{1+C_{1} z} \leq \frac{C_{2} z}{1+C_{2} z}
$$

So, the supremum $C$ must be found. There are four cases:

$$
\begin{aligned}
& \text { 1. } a \leq 0, b \leq 0, \text { sup } c=A^{4} B^{4} \text {, so } \Delta_{s}=z A^{4} B^{4} /\left(1+z A^{4} B^{4}\right) \\
& \text { 2. } a>0, b>0, \text { sup } c=1 \text {, so } \Delta_{s}=z /(1+z) \\
& \text { 3. } a>0, b \leq 0, \text { sup } c=B^{4} \text {, so } \Delta_{s}=B^{4} z /\left(1+B^{4} z\right) \\
& \text { 4. } a \leq 0, b>0, \text { sup } c=A^{4} \text {, so } \Delta_{s}=A^{4} z /\left(1+A^{4} z\right) .
\end{aligned}
$$

Since $2 z C /(1+z C)$ must be less than 1 , it is immediately established that $\mu<0$. For the four cases:

$$
\begin{aligned}
& \text { 1. } \mu<4 a+4 b \\
& \text { 2. } \mu<0 \\
& \text { 3. } \mu<4 b \\
& \text { 4. } \mu<4 a
\end{aligned}
$$

A similar set of statements can be made for $s=10$. Consider now the 
case $s=1(2,3,4): \quad \Delta_{s}^{C} \leq \Delta_{s}^{C}$ implies that

$$
\frac{\mathrm{C}_{1} z|\mathrm{~B}-1|}{\left(1+\mathrm{C}_{1} z\right)\left(1+\mathrm{C}_{z B} \mathrm{~B}\right)} \leq \frac{\mathrm{C}_{2} z|\mathrm{~B}-1|}{\left(1+\mathrm{C}_{2} z\right)\left(1+\mathrm{C}_{2} z \mathrm{~B}\right)} \text {, }
$$

which implies that $\mathrm{C}_{1}-\mathrm{C}_{2} \leq \mathrm{Bz}^{2} \mathrm{C}_{1} \mathrm{C}_{2}$. There are two possibilities:

(1) $C_{1} \geq C_{2}$, which implies that $1 \leq \mathrm{Bz}^{2} \mathrm{C}_{1} \mathrm{C}_{2}$. So, sup $\Delta_{s}$ comes from the minimum $C$, which is for the four cases:
1. 1
2. $A^{4} B^{3}$
3. $A^{4}$
4. $\mathrm{B}^{3}$.

It can be observed at this point that in each of the four cases that the inequality in (1) cannot be satisfled:

$$
\begin{aligned}
& \text { 1. } \mathrm{z}^{2}<\mathrm{e}^{2 \beta(4 a+4 b)}=\mathrm{A}^{-8} \mathrm{~B}^{-8}, \mathrm{BC}_{1} \mathrm{C}_{2}<\mathrm{A}^{8} \mathrm{~B}^{8}, \text { or } \mathrm{Bz}^{2} \mathrm{C}_{1} \mathrm{C}_{2}<1 . \\
& \text { 2. } \mathrm{z}^{2}<1, \mathrm{BC}_{1} \mathrm{C}_{2}<1 \text {, or } \mathrm{Bz}^{2} \mathrm{C}_{1} \mathrm{C}_{2}<1 \text {. } \\
& \text { 3. } \mathrm{z}^{2}<\mathrm{e}^{2 \beta(4 \mathrm{~b})}=\mathrm{B}^{-8}, \mathrm{BC}_{1} \mathrm{C}_{2}<\mathrm{B}^{8} \text {, or } \mathrm{Bz}^{2} \mathrm{C}_{1} \mathrm{C}_{2}<1 . \\
& \text { 4. } \mathrm{z}^{2}<\mathrm{e}^{2 \beta(4 a)}=\mathrm{A}^{-8}, \mathrm{BC}_{1} \mathrm{C}_{2} \leq \mathrm{A}^{8} \text {, or } \mathrm{Bz}^{2} \mathrm{C}_{1} \mathrm{C}_{2}<1 .
\end{aligned}
$$

So, the other possibility must be considered.

(2) $\mathrm{C}_{1}<\mathrm{C}_{2}$, which implies that $1 \geq \mathrm{Bz}^{2} \mathrm{C}_{1} \mathrm{C}_{2}$, so sup $\Delta_{s}$ comes from the maximum $C$. For the four cases sup $C$ is given by

$$
\begin{aligned}
& \text { 1. } A^{4} B^{3} \\
& \text { 2. } 1 \\
& \text { 3. } B^{3} \\
& \text { 4. } A^{4} .
\end{aligned}
$$

For the four cases $\Delta_{S}$ can be written down

$$
\begin{aligned}
& \text { 1. } \sup \Delta_{S}=z A^{4} B^{3}(B-1) /\left(1+A^{4} B^{3} z\right)\left(1+A^{4} B^{4} z\right) \\
& \text { 2. } \sup \Delta_{S}=z(1-B) /(1+z)(1+B z)
\end{aligned}
$$




$$
\begin{aligned}
& \text { 3. sup } \Delta_{S}=2 B^{3}(B-1) /\left(1+B^{3} z\right)\left(1+B^{4} z\right) \\
& \text { 4. sup } \Delta_{S}=2 A^{4}(1-B) /\left(1+A^{4} z\right)\left(1+A^{4} B z\right) \text {. }
\end{aligned}
$$

In completely analagous manner, for $s=5(6,7,8)$ :

$$
\begin{aligned}
& \text { 1. sup } \Delta_{S}=z A^{3} B^{4}(A-1) /\left(1+A^{3} B^{4} z\right)\left(1+A^{4} B^{4} z\right) \\
& \text { 2. sup } \Delta_{S}=z(1-A) /(1+z)(1+A z) \\
& \text { 3. sup } \Delta_{S}=z B^{4}(1-A) /\left(1+B^{4} z\right)\left(1+B^{4} A z\right) \\
& \text { 4. } \sup \Delta_{S}=z A^{3}(A-1) /\left(1+A^{3} z\right)\left(1+A^{4} z\right) .
\end{aligned}
$$

It is now possible to write down the Dobrushin uniqueness criterion for the four cases:

$$
\begin{aligned}
& \frac{4 z A^{4} B^{3}(B-1)}{\left(1+A^{4} B^{3} z\right)\left(1+A^{4} B^{4} z\right)}+\frac{4 z A^{3} B^{4}(A-1)}{\left(1+A^{3} B^{4} z\right)\left(1+A^{4} B^{4} z\right)}+\frac{2 z A^{4} B^{4}}{1+z A^{4} B^{4}}<1 \\
& \frac{4 z(1-B)}{(1+z)(1+z B)}+\frac{4 z(1-A)}{(1+z)(1+A z)}+\frac{2 z}{1+z}<1 \\
& \frac{4 z B^{3}(B-1)}{\left(1+B^{3} z\right)\left(1+B^{4} z\right)}+\frac{4 z B^{4}(1-A)}{\left(1+B^{4} z\right)\left(1+B^{4} A z\right)}+\frac{2 B^{4} z}{1+B^{4} z}<1 \\
& \frac{4 z A^{4}(1-B)}{\left(1+A^{4} z\right)\left(1+A^{4} B z\right)}+\frac{4 z A^{3}(A-1)}{\left(1+A^{3} z\right)\left(1+A^{4} z\right)}+\frac{2 A^{4} z}{1+A^{4} z}<1 .
\end{aligned}
$$

Typical regions of uniqueness are shown in Figures 10, 11, and 12. c. High and Low $\beta$ Limits of the Uniqueness Region for the Restricted Dimer Mode1. It is relatively easy to see that the last expressions derived in Paragraph $B$ of this Section are dominated by

$$
\begin{aligned}
& \text { 1. } 10 z A^{4} B^{4} /\left(1+z A^{4} B^{4}\right) \\
& \text { 2. } 10 z /(1+z) \\
& \text { 3. } 10 z B^{4} /\left(1+B^{4} z\right) \\
& \text { 4. } 10 z A^{4} /\left(1+A^{4} z\right) .
\end{aligned}
$$

If these expressions are made to be less than 1 , the original expressions certainly will be. All of this implies

$$
\text { 1. } \mu<-\log 9 / \beta+4 a+4 b
$$




$$
\begin{aligned}
& \text { 2. } \mu<-\log 9 / \beta \\
& \text { 3. } \mu<-\log 9 / \beta+4 b \\
& \text { 4. } \mu<-\log 9 / \beta+4 a
\end{aligned}
$$

Clearly, these are also the high $\beta$ limits on the uniqueness region boundary.

A first order expansion in $\beta$ gives the following low $\beta$ limits on $\mu$ for uniqueness:

$$
\begin{aligned}
& \text { 1. } \mu<6 a+6 b \\
& \text { 2. } \mu<-2(a+b) \\
& \text { 3. } \mu<6 a-2 b \\
& \text { 4. } \mu<-2 a+6 b .
\end{aligned}
$$

This suggests that there may be a lower bound on the boundary of the uniqueness condition. This turns out to be the case.

1. As sume: $\mu<6(a+b)$, or $z<A^{-6} B^{-6}, A \geq 1, B \geq 1$. Then,

$$
\begin{aligned}
\partial \Delta= & \frac{A^{4} B^{3}\left[B\left(1+A^{4} B^{3} z\right)^{2}+4(B-1)\left(1-A^{8} B^{7} z^{2}\right)\right]}{\left(1+A^{4} B^{4} z\right)^{2}\left(1+A^{4} B^{3} z\right)^{2}}+ \\
& \frac{A^{3} B^{4}\left[A\left(1+A^{3} B^{4} z\right)^{2}+4(A-1)\left(1-A^{7} B^{8} z^{2}\right)\right]}{\left(1+A^{4} B^{4} z\right)^{2}\left(1+A^{3} B^{4} z\right)^{2}} \geq 0 .
\end{aligned}
$$

For maximum $z, \Delta<4 /\left(1+A^{-2} B^{-3}\right)-4 /\left(1+A^{-2} B^{-2}\right)+4 /\left(1+A^{-3} B^{-2}\right)-$

$$
4 /\left(1+A^{-2} B^{-2}\right)=\Delta_{m} \text {. }
$$

Then $\frac{\partial A_{m}}{\partial A}=\frac{12 A^{-3} B^{-2}\left(A^{-1}-1\right)\left(1-A^{-5} B^{-4}\right)}{\left(1+A^{-2} B^{-3}\right)^{2}\left(1+A^{-2} B^{-2}\right)^{2}} \leq 0$,

$$
\frac{\partial \Delta_{m}}{\partial B}=\frac{12 B^{-3} A^{-2}\left(B^{-1}-1\right)\left(1-A^{-4} B^{-5}\right)}{\left(1+A^{-3} B^{-2}\right)^{2}\left(1+A^{-2} B^{-2}\right)^{2}} \leq 0,
$$

from which it is concluded that for $\mu<6(a+b)$ the Gibbsian distribution is unique, since $\Delta_{\mathrm{m}}$ is a maximum for $A=B=1$ where $\Delta_{\mathrm{m}}=1$, so $\Delta<1$. 
2. Assume: $\mu<-2(a+b)$, or $z<A^{2} B^{2}, A \leq 1, B \leq 1$.

Then $\frac{\partial \Delta}{\partial z}=\frac{(1+B z)^{2}+4(1-B)\left(1-B z^{2}\right)}{(1+B z)^{2}(1+z)^{2}}+\frac{(1+A z)^{2}+4(1-A)\left(1-A z^{2}\right)}{(1+A z)^{2}(1+z)^{2}} \geq 0$.

For maximum $z, \Delta<4 /\left(1+A^{2} B^{3}\right)-4 /\left(1+A^{2} B^{2}\right)+4 /\left(1+A^{3} B^{2}\right)$

$$
-4 /\left(1+A^{2} B^{2}\right)+2 A^{2} B^{2} /\left(1+A^{2} B^{2}\right)=4_{m}
$$

Then $\frac{\partial \Delta_{m}}{\partial A}=\frac{8 A B^{2}(1-B)\left(1-A^{4} B^{5}\right)}{\left(1+A^{2} B^{2}\right)^{2}\left(1+A^{2} B^{3}\right)^{2}}+\frac{12 A B^{2}(1-A)\left(1-A^{5} B^{4}\right)}{\left(1+A^{2} B^{2}\right)^{2}\left(1+A^{3} B^{2}\right)^{2}} \geq 0$,

and $\frac{\partial \Delta_{m}}{\partial B}=\frac{8 A^{2} B(1-A)\left(1-A^{5} B^{4}\right)}{\left(1+A^{2} B^{2}\right)^{2}\left(1+A^{3} B^{2}\right)^{2}}+\frac{12 A^{2} B(1-B)\left(1-A^{4} B^{5}\right)}{\left(1+A^{2} B^{2}\right)^{2}\left(1+A^{2} B^{3}\right)^{2}} \geq 0$.

from which it is concluded that for $\mu<-2(a+b)$ the Gibbsian distribution is unique, since $\Delta_{m}$ is a maximum for $A=B=1$, where $\Delta_{m}=1$. So, $\Delta<1$.

3. Assume: $\mu<6 b-2 a, z<B^{-6} A^{2}, A \leq 1, B \geq 1$.

$$
\text { Then } \begin{aligned}
\frac{\partial \Delta}{\partial z}= & \frac{B^{3}\left[B\left(1+B^{3} z\right)^{2}+4(B-1)\left(1-B^{7} z^{2}\right)\right]}{\left(1+B^{3} z\right)^{2}\left(1+B^{4} z\right)^{2}} \\
& +\frac{B^{4}\left[\left(1+A B^{4} z\right)^{2}+4(1-A)\left(1-z^{2} A B^{8}\right)\right]}{\left(1+B^{4} z\right)^{2}\left(1+A B^{4} z\right)^{2}} \geq 0 .
\end{aligned}
$$

For maximum $z, \Delta<4 /\left(1+B^{-3} A^{2}\right)-4 /\left(1+A^{2} B^{-2}\right)+4 /\left(1+A^{3} B^{-2}\right)$

$$
-4 /\left(1+A^{2} B^{-2}\right)+2 A^{2} B^{-2} /\left(1+A^{2} B^{-2}\right)=\Delta_{m} \text {. }
$$

Then $\frac{\partial \Delta_{m}}{\partial A}=\frac{8 A B^{-2}\left(1-B^{-1}\right)\left(1-A^{4} B^{-5}\right)}{\left(1+B^{-3} A^{2}\right)^{2}\left(1+A^{2} B^{-2}\right)^{2}}+\frac{12 A B^{-2}(1-A)\left(1-A^{5} B^{-4}\right)}{\left(1+A^{3} B^{-2}\right)^{2}\left(1+A^{2} B^{-2}\right)^{2}} \geq 0$,

and $\frac{\partial \Delta_{m}}{\partial B}=\frac{12 A^{2} B^{-3}\left(B^{-1}-1\right)\left(1-A^{4} B^{-5}\right)}{\left(1+B^{-3} A^{2}\right)^{2}\left(1+A^{2} B^{-2}\right)^{2}}+\frac{8 A^{2} B^{-2}(A-1)\left(1-A^{5} B^{-4}\right)}{\left(1+A^{3} B^{-2}\right)^{2}\left(1+A^{2} B^{-2}\right)^{2}} \leq 0$,

from which it is concluded that for $\mu<6 b-2 a$ the Gibbsian distribution is unique, since $\Delta_{m}$ is a maximum for $A=B=1$ where $\Delta_{m}=1$, so $\Delta<1$.

4. For $\mu<6 a-2 b, A \geq 1, B \leq 1, \Delta<1$, by symmetry with Case 3 . 
IV. NON-UNIQUENESS REGION IN $\beta-\mu$ HALF-PLANE.

A. The General Contour. Method. Represent a vertical dimer's reference site by $a+$ and a horizontal dimer's reference site by a $o$. Unoccupied reference sites are represented by dots, and the other square lattice sites are deleted to avoid clutter. The nematic and smectic boundary conditions are illustrated in Figures 6 and 7 . The interior region will be denoted V. Contours are defined for a particular configuration in $\mathrm{V}$ by adding molecules from the 'outside in' from the distant boundary. The added dimers must conform to the close-packed structure associated with the boundary, and each added dimer must be adjacent to at least one other of a chain of these ordered dimers extending to the boundary of $\mathrm{V}$. The dimers are added until no more dimers can be added without interfering with a dimer in $V$. These added dimers define the outer contours (boundaries) of the disordered phase and are removed after the contours have been drawn. Examples are given in Figures 6 and 7. Every contour defined in this way is an 'outer contour.' Inner contours are not defined or needed for this problem. Note that for any configuration in $\mathrm{V}$ no two outer contours intersect: in fact, there must be room to place close-packed ordered nematic (smectic) dimers between any two outer contours. Let $G$ be a particular outer contour and $|G|$ be the length of the contour in lattice parameter units. The energy of a particular configuration $\alpha$ in $V$ is

$$
\begin{aligned}
\mathrm{U}_{(\alpha)}= & \mathrm{U}_{\mathrm{I}}+\mathrm{U}_{\mathrm{II}}+\mathrm{U}_{\mathrm{III}} \text {, where } \\
\mathrm{U}_{\mathrm{I}}= & \text { self energy of dimers inside } \mathrm{G} \text { and interactions with each } \\
& \text { other } \\
\mathrm{U}_{\mathrm{II}}= & \text { self energy of dimers outside } \mathrm{G} \text { and interactions with each } \\
& \text { other }
\end{aligned}
$$


$\mathrm{U}_{\text {III }}=$ interaction on dimers inside $G$ with those outside $G$.

The self energy of a dimer is the negative of the chemical potential.

The probability of the contour $G, P(G)$, is given by (10)

$$
\begin{aligned}
P(G)=\sum_{\alpha \beta G} \exp (-\beta U(\alpha)) / & \sum \exp (-\beta U(\alpha)), \\
& \text { a } 11 \alpha \\
& \text { in } V
\end{aligned}
$$

where $\alpha \mathcal{G}^{G}$ stands for those configurations inside $V$ which contain $G$. An upper bound will be derived for $P(G)$ that will depend only on $|G|$, by the standard technique of underestimating the denominator in the equation for $P(G)$. Consider a configuration transformation $T$ that operates on any configuration $\alpha$, defined as follows:

a) Inside $\mathrm{G}$ a horizontal dimer at $(\mathrm{x}, \mathrm{y})$ becomes a vertical dimer at $(\mathrm{x}, \mathrm{y})$.

b) Inside $G$ a vertical dimer at $(x, y)$ becomes a horizontal dimer at $(\mathrm{x}-1, \mathrm{y}+1)$

c) After a) and b) fill in, where possible, dimers one layer inside and outside of $G$. The added dimers must follow the same rules as imagined to be added to define the contour initially. Figures 8 and 9 give examples of the full $T$ transformation.

Step c) means that $T$ is not a one-to-one transformation; however, the degeneracy of the transformation is certainly less than $4|G|$. This is because there are only four combinations of dimer configurations for each piece of contour (outside contour piece occupied or unoccupied; inside contour piece occupied or unoccupled.) Define $G_{a}\left(G_{b}\right)$ as the length of that portion of $G$ which has an $a(b)$ interaction across it after T. These definitions are unique.

B. Negative Pair Interactions. Note that $\mathbf{T}$ preserves the interactions of the dimers in $\alpha$ inside $G$ with each other. It also preserves the in- 
teractions of the dimers in $\alpha$ outside $G$ with each other. In addition $T$ may introduce negative terms to $U_{I}$ by the interaction of any mo1ecules added inside $G$ by $T$ with those dimers inside $G$ which were roby $T$. T may introduce a negative contribution to $U_{I I}$ by the interactions of any molecules added just outside $G$ with the other molecules outside G. If the chemical potential is positive (negative), $T$ introduces a negative (positive) contribution to $U_{I}$ and $\nabla_{I I}$. The crucial point in the proof is the analysis of the change in $U_{\text {III }}$ after $T . T$ was designed to obtain the close-packed interaction across the contour G. So, after $T$ one has an ' $a$ ' interaction across a $G_{a}$ contour plece and $a{ }^{\prime} b$ ' interaction across $a G_{b}$ piece.

Examination of the configuration $\alpha$ before $T$ reveals that the contour energy cannot have any of the close-packed interactions (by definition of the contour.) In the nematic case, there cannot be any ' $a$ ' interactions. (There are no horizontal dimers just outside G.) However, It is possible to have $a$ ' $b$ ' interaction across practically every plece of contour length ( $|G|-2$ to exact.) In the smectic case before $T$ there can be no ' $b$ ' interactions across the boundary since there cannot be a reference site in the right place. However, it is possible to have an ' $a$ ' interaction across $a G_{b}$ contour piece. It can be shown that for each such ' $a$ ' interaction across $a G_{b}$ contour piece there is an immediately adjacent $G_{b}$ contour piece across which it is impossible to have any interaction. It can be observed that it is impossible in the nematic (smectic) case to traverse two connected $G_{b}\left(G_{a}\right)$ steps consecutively. Hence,

$$
\begin{aligned}
& G_{a} \geq|G| / 2, \text { nematic case. } \\
& G_{b} \geq|G| / 2, \text { smectic case. }
\end{aligned}
$$


The following relations can be written down. Note that in the smectic equation the fact that $b-(1 / 2) a$ is negative is used, and $T_{\alpha}$ is the configuration $\alpha$ transformed by $T$.

$$
\begin{aligned}
& \mathrm{U}_{\mathrm{I}}\left(\mathrm{T}_{\alpha}\right) \leq \mathrm{U}_{\mathrm{I}}(\alpha), \mu \geq 0 \text {. } \\
& \mathrm{U}_{\mathrm{I}}\left(\mathrm{T}_{\alpha}\right) \leq \mathrm{U}_{\mathrm{I}}(\alpha)-\mu|\mathrm{G}|, \mu<0 \text {. } \\
& \mathrm{U}_{\mathrm{II}}\left(\mathrm{T}_{\alpha}\right) \leq \mathrm{U}_{\mathrm{II}}(\alpha), \mu \geq 0 \text {. } \\
& \mathrm{U}_{\mathrm{II}}\left(\mathrm{T}_{\alpha}\right) \leq \mathrm{U}_{\mathrm{II}}(\alpha)-\mu|\mathrm{G}|, \mu<0 \text {. } \\
& \mathrm{U}_{\mathrm{III}}\left(\mathrm{T}_{\alpha}\right)=\mathrm{G}_{\mathrm{a}} \mathrm{a} \text {, nematic case. } \\
& \mathrm{U}_{\mathrm{III}}\left(\mathrm{T}_{\alpha}\right)=\mathrm{G}_{\mathrm{b}} \mathrm{b}+\mathrm{G}_{\mathrm{a}} \mathrm{a} \text {, smectic case. } \\
& \mathrm{U}_{\text {III }}(\alpha) \geq|\mathrm{G}| \mathrm{b} \text {, nematic case. } \\
& \mathrm{U}_{\operatorname{III}}(\alpha) \geq(1 / 2) \mathrm{G}_{\mathrm{b}} \mathrm{a} \text {, smectic case. }
\end{aligned}
$$

It can then be concluded that the transformation produced the following changes:

$$
\begin{aligned}
& \mathrm{U}\left(\mathrm{T}_{\alpha}\right)-\mathrm{U}(\alpha) \leq|\mathrm{G}|((1 / 2) \mathrm{a}-\mathrm{b}) \text {, nematic case, } \mu \geq 0 . \\
& \mathrm{U}\left(\mathrm{T}_{\alpha}\right)-\mathrm{U}(\alpha) \leq|\mathrm{G}|\left((1 / 2) \mathrm{a}-\mathrm{b}-\mathrm{2}_{\mu}\right) \text {, nematic case, } \mu<0 . \\
& \mathrm{U}\left(\mathrm{T}_{\alpha}\right)-\mathrm{U}\left({ }_{\alpha}\right) \leq(1 / 2)|G|(\mathrm{b}-(1 / 2) \mathrm{a}) \text {, smectic case, } \mu \geq 0 . \\
& \mathrm{U}\left(\mathrm{T}_{\alpha}\right)-\mathrm{U}(\alpha) \leq(1 / 2)|G|(\mathrm{b}-(1 / 2) \mathrm{a}-4 \mu) \text {, smectic case, } \mu<0 .
\end{aligned}
$$

If only those configurations derived from $T$ are used in the sum in the donominator of the expression for $P(G)$, the probability of $G$ occuring can be estimated:

$$
\begin{aligned}
& P(G) \leq 4|G| \exp (\beta|G|((1 / 2) a-b)) \text {, nematic case, } \mu \geq 0 \\
& P(G) \leq 4|G| \exp (\beta|G|((1 / 2) a-b-2 \mu)) \text {, nematic case, } \mu<0 \\
& P(G) \leq 4|G| \exp ((1 / 2) \beta|G|(b-(1 / 2) a)) \text {, smectic case, } \mu \geq 0 . \\
& P(G) \leq 4|G| \exp ((1 / 2) \beta|G|(b-(1 / 2) a-4 \mu)) \text {, smectic case, } \mu<0 .
\end{aligned}
$$

The number density $\rho_{\mathrm{d}}$ of the disordered dimers (those not orienting themselves according to the boundary conditions to $v$ ) is bounded by 


$$
\rho_{\mathrm{d}} \leq \sum_{|G|=0}^{\infty} \mathrm{P}(|\mathrm{G}|) \mathrm{K}(|\mathrm{G}|)
$$

where $\mathrm{K}(|\mathrm{G}|)$ is the modified Peier1's upper bound on the number of contours (closed) on the square lattice of length $|G|$ around any specified site $(13,14)$.

$$
K(|G|)=(1 / 36) 3^{|G|}|G| \text {, }
$$

and $P(|G|)$ is the upper bound to the probability of a contour of length $|G|$ derived above. The number density bound can be written as

$$
\rho_{\mathrm{d}} \leq(1 / 36) \stackrel{\infty}{\sum_{|G|=0}}|G| \exp (|G|(\log 12+\beta X)) .
$$

The value for $\mathrm{X}$ is for the various cases is

$$
\begin{aligned}
& (1 / 2) a-b, \text { nematic, } \mu \geq 0 . \\
& (1 / 2) a-b-2 \mu, \text { nematic, } \mu<0 . \\
& (1 / 2) b-(1 / 4) a, \text { smectic, } \mu \geq 0 . \\
& (1 / 2) b-(1 / 4) a-2 \mu, \text { smectic, } \mu<0 .
\end{aligned}
$$

If $X$ is negative, the bound on $\rho_{d}$ can be made arbitrarily small for sufficiently large $\beta$. Specifically, it can be made less that $1 / 2$. The same arguments can be repeated for a1tered boundary conditions. For example, in the nematic case change all of the boundary vertical nematic dimers shown in Figure 6 to close-packed nematic structure horizontally packed. Whereas before in the nematic case it was shown that the probability of a horizontally aligned dimer could be made arbitrar11y sma11, now the same thing can be said about the probability of a vertically aligned dimer. In other words, the alignment probability depends upon the boundary conditions, assuming $X<0$ and the temperature sufficiently sma11, of course. It should also be noted that this proof is independent of the size of $V$, so that what is being demonstrated is Indeed long range order even though the interactions are short range. 
C. Predominant1y Negative Pair Interactions. It was shown in the previous paragraph that long range order could exist for low enough temperature for $a$ and $b$ negative with the possible exception of $a=2 b$. It is not unreasonable to expect that nematic (smectic) boundary conditions could show that there is long range order for positive $b$ (a) provided a (b) is sufficiently negative. Indeed, this proves to be the case. The principal observation that must be made is that the dimers added by T may add a positive interaction term which cannot be larger than $4 \mathrm{~b}$ (4a) per unit contour length for the nematic (smectic) case since this is $a 11$ the ' $a$ ' or ' $b$ ' interactions that $a$ dimer can accept regardless of the sign of $a$ or $b$. As before, a conservative estimate can also be made that no more than two dimers can be added per unit contour length (one inside and one outside G).

The energy relations can be written down for the nematic case $(a<0$, b>0, nematic boundary conditions):

$$
\begin{aligned}
& \mathrm{U}_{\mathrm{I}}\left(\mathrm{T}_{\alpha}\right) \leq \mathrm{U}_{\mathrm{I}}(\alpha)+4 \mathrm{~b}|\mathrm{G}|, \mu \geq 0 . \\
& \mathrm{U}_{\mathrm{I}}\left(\mathrm{T}_{\alpha}\right) \leq \mathrm{U}_{\mathrm{I}}(\alpha)+4 \mathrm{~b}|\mathrm{G}|-\mu|\mathrm{G}|, \mu<0 . \\
& \mathrm{U}_{\mathrm{II}}\left(\mathrm{T}_{\alpha}\right) \leq \mathrm{U}_{\mathrm{II}}(\alpha)+4 \mathrm{~b}|\mathrm{G}|, \mu \geq 0 . \\
& \mathrm{U}_{\mathrm{II}}\left(\mathrm{T}_{\alpha}\right) \leq \mathrm{U}_{\mathrm{II}}(\alpha)+4 \mathrm{~b}|\mathrm{G}|, \mu<0 . \\
& \mathrm{U}_{\mathrm{III}}(\alpha) \geq 0 . \\
& \mathrm{U}_{\mathrm{III}}\left(\mathrm{T}_{\alpha}\right)=\mathrm{aG} \mathrm{a} \leq(1 / 2) \mathrm{a}|\mathrm{G}| . \\
& \mathrm{U}\left(\mathrm{T}_{\alpha}\right)-\mathrm{U}\left({ }_{\nu}\right) \leq((1 / 2) \mathrm{a}+8 \mathrm{~b})|\mathrm{G}|, \mu \geq 0 . \\
& \mathrm{U}\left(\mathrm{T}_{\alpha}\right)-\mathrm{U}(\alpha) \leq((1 / 2) \mathrm{a}+8 \mathrm{~b}-2 \mu)|\mathrm{G}|, \mu<0 .
\end{aligned}
$$

The same arguments can be made as before in the preceeding paragraph of this section, the requirement being that $\mathrm{x}<0$, where $\mathrm{X}$ is

$$
\begin{aligned}
& (1 / 2) a+8 b, \mu \geq 0 . \\
& (1 / 2) a+8 b-2 \mu, \mu<0 .
\end{aligned}
$$


The energy relations can also be written down for the smectic case ( $b<0, a>0$, smectic boundary conditions):

$$
\begin{aligned}
& U_{I}\left(T_{\alpha}\right)-U_{I}(\alpha) \leq 4 a|G|, \mu \geq 0 . \\
& U_{I}\left(T_{\alpha}\right)-U_{I}(\alpha) \leq 4 a|G|-\mu|G|, \mu<0 . \\
& U_{I I}\left(T_{\alpha}\right)-U_{I I}(\alpha) \leq 4 a|G|, \mu \geq 0 . \\
& U_{I I}\left(T_{\alpha}\right)-U_{I I}(\alpha) \leq 4 a|G|-\mu|G|, \mu<0 . \\
& U_{I I I}(\alpha) \geq 0 . \\
& U_{I I I}\left(T_{\alpha}\right)=b G_{b}+a G a \leq(1 / 2)(a+b)|G| . \\
& U\left(T_{\alpha}\right)-U(\alpha) \leq(1 / 2)(b+17 a)|G|, \mu \geq 0 . \\
& U\left(T_{\alpha}\right)-U(\alpha) \leq(1 / 2)(b+17 a-4 \mu)|G|, \mu<0 .
\end{aligned}
$$

The same arguments can be made as before as in the preceeding paragraph of this section, the requirement being that $x<0$, where in this case $X$ is

$$
\begin{aligned}
& (1 / 2)(b+17 a), \mu \geq 0 \\
& (1 / 2)(b+17 a-4 \mu), \mu<0 .
\end{aligned}
$$

D. Summing the Number Density Series. Recall that the number density series was given by

$$
\rho_{\mathrm{d}} \leq(1 / 36) \sum_{|G|=0}^{\infty}|G| \exp ((\log (12)+\beta X)|G|)
$$

Let $Y=\exp (\log (12)+\beta X), X<0$ : then this series converges if $\beta$ is large enough. So,

$$
\rho_{\mathrm{d}} \leq(1 / 36) \sum_{|G|=0}^{\infty}|G| Y|G|=(1 / 36) Y /(1-Y)^{2}, 0 \leq Y<1 .
$$

To show long range order it is necessary to show that $\rho_{d}$ is less than (1/2). This is true if

$$
(1 / 36) \mathrm{Y} /(1-\mathrm{Y})^{2}<1 / 2
$$

This relation is satisfied if $\mathrm{Y}<\mathrm{Y}_{0}$, where

$$
\mathrm{Y}_{0}=(37 / 36)(1-\sqrt{73} / 37)
$$


which implies that

$\beta>\log \left(\mathrm{Y}_{\mathrm{O}} / 12\right) / \mathrm{X} \approx-2.72 / \mathrm{X}$

This gives the desired lower bound on $\beta$ for non-uniqueness when $\mathrm{x}<0$. 
V. PERTURBATION CALCULATION--LOW TEMPERATURE LIMIT.

A. Assumptions. It would be useful to know for what $\mu$ the random phase and the ordered phase coexist at low temperatures. The following assumptions are made. This type of equilibrium is assumed at low temperatures (15).

low density gas $\underset{\leftarrow}{\rightarrow}$ high density solid.

The low density phase is assumed an Ideal lattice gas with volume=B= number of sites on the restricted square lattice. The number of 1attice sites available to the dimer is $C=2 B=$ number of sites on the auxiliary lattice. For the low density gas, $E=0$, and $\mathrm{S}=\mathrm{k} \log \left(\begin{array}{l}\mathrm{C} \\ \mathrm{g}\end{array}\right)$, where $\mathrm{N}_{\mathrm{g}}$ is the number of dimers. The free energy is

$$
\begin{aligned}
\text { E-TS } & =-k T \log \left(\begin{array}{l}
2 B \\
N
\end{array}\right)=-k T \log \left[(2 B) ! /\left(\left(2 B-N_{g}\right) !\left(N_{g}\right) !\right)\right] \\
& =2 B k T\left[\rho_{g} \log _{g}+\left(1-\rho_{g}\right) \log \left(1-\rho_{g}\right)\right],
\end{aligned}
$$

using Stirling's approximation and $\mathrm{N}_{\mathrm{g}}=2 \mathrm{~B} \rho_{\mathrm{g}}$, where $\rho_{\mathrm{g}}$ is the gas density. The pressure $\mathrm{P}_{\mathrm{g}}$ is given by $-\left(\frac{\partial A}{\partial \mathrm{B}}\right)_{\mathrm{T}, \mathrm{N}_{\mathrm{g}}}$. The chemical potential is given by $\left(\frac{\partial A}{\partial N}\right)_{T}, B$ So,

$$
\begin{aligned}
& P_{g}=-2 k T \log \left(1-\rho_{g}\right) \\
& \mu_{g}=k T \log \left(\rho_{g} /\left(1-\rho_{g}\right)\right) .
\end{aligned}
$$

For the high density solid phase at low temperature, almost a11 of the sites of the restricted square lattice will be filled, and $S=k \log \left(\begin{array}{l}B \\ N\end{array}\right)$, where $\mathrm{N}_{s}$ is the number of dimers in $B$. Note also that

$$
\begin{aligned}
E & =2 a B-\left(B-N_{s}\right) 4 a, \text { for nematic close-packed structure. } \\
E & =B(a+2 b)-\left(B-N_{s}\right)(2 a+4 b), \text { for smectic close-packed structure. } \\
A(\text { nematic }) & =2 a\left(2 N_{s}-B\right)-k T N_{s} \\
& =2 a\left(2 N_{s}-B\right)+k T B\left[2 \rho_{s} \log \left(2 \rho_{s}\right)+\left(1-2 \rho_{s}\right) \log \left(1-2 \rho_{s}\right)\right]
\end{aligned}
$$


using Stirling's Approximation and $2 B \rho_{s}=N_{s}$. Hence,

$$
\begin{aligned}
& P_{s}=2 a-k T \log \left(1-2 p_{s}\right) . \\
& \mu_{s}=4 a+k T \log \left[\left(2 p_{s}\right) /\left(1-2 p_{s}\right)\right] .
\end{aligned}
$$

Similarly, for the smectic case

$$
\begin{aligned}
P_{s} & =2 b+a-k T \log \left(1-2 p_{s}\right) \\
\mu_{s} & =2(a+2 b)+k T \log \left[\left(2 p_{s}\right) /\left(1-2 p_{s}\right)\right] .
\end{aligned}
$$

To summarlze, for the low density gas

$$
\begin{aligned}
P_{g} & =-2 k T \log \left(1-\rho_{g}\right) . \\
\mu_{g} & =k T \log \left[\rho_{g} /\left(1-\rho_{g}\right)\right] .
\end{aligned}
$$

For the high density solid phase

$$
\begin{aligned}
& P_{s}=2 a-k T \log \left(1-2 \rho_{s}\right), \\
& \mu_{s}=4 a+k T \log \left[\left(2 p_{s}\right) /\left(1-2 \rho_{s}\right)\right], \text { nematic structure. } \\
& P_{s}=(a+2 b)-k T \log \left(1-2 \rho_{s}\right), \\
& \mu_{s}=2(a+2 b)+k T \log \left[\left(2 \rho_{s}\right) /\left(1-2 \rho_{s}\right)\right], \text { smectic structure. }
\end{aligned}
$$

B. Perturbation Calculation. A perturbation can be done which is zeroth order in $\rho_{\mathrm{g}}$ and $1-2 \rho_{\mathrm{s}}$. At equilibrium the pressures and chemical potentials for each phase are equated. In the nematic case:

$$
\begin{aligned}
& 0=2 a-k T \log \left(1-2 \rho_{s}\right) \\
& k T \log \rho_{g}=4 a+k T \log \left(1-2 \rho_{s}\right) .
\end{aligned}
$$

These equations are easily solved to yield

$$
\begin{aligned}
& \rho_{g}=1-2 \rho_{s}=\exp (2 a / k T), \\
& P=2 k \exp (2 a / k T), \\
& \mu=2 a .
\end{aligned}
$$

Similarly, for the smectic case

$$
\begin{aligned}
& P=2 k \operatorname{Texp}((a+2 b) / k T), \\
& \mu=a+2 b .
\end{aligned}
$$

It can be concluded then that if the two phases coexist at low temper- 
atures that the chemical potential for which they do so is the average close-packed energy of the solid structure. 
VI. SOME SPECIFIC EXAMPIES.

A. Predominantly Negative Para1le1 Interactions. Assume that $a=4 b<0$. The regions of uniqueness and non-uniqueness as derived in Sections III and IV are indicated in Figure 10. The region of analyticity as derived by the YLR Theorem is not shown since the maximum $\beta$ of this region is a factor of about $10^{5}$ less than that of the minimum $\beta$ derived for the non-uniqueness region. Nevertheless, it is important to remember that the analytic region (presumably representing the random phase) extends a11 the way to $\mu=+\infty$. Hence, the dashed IIne in Figure 10 which stands for the presumed phase coexistence interface goes to to. For negative $\mu$, there exists regions of uniqueness (short-range Independence) and non-uniqueness (1ong-range order), so it is natural to assume that there is a line between the regions where the two phases coexist. This line is drawn asymptotic to the line $\mu=2 a=8 b$ as derived by the perturbation method in Section $V$. It is not known whether or not the phase-coexistence line goes to $\beta=0$ as $\mu-\infty$ or levels off to some horizontal positive $\beta$ asymptote. (i.e., is there a critical temperature?) Other graphs in the $\beta-\mu$ plane of these regions with $a<2 b$ look quite similar even with $b$ slightly positive $(a<-16 b)$. B. Predominantly Negative Perpendicular Interactions. Assume $a=b<0$. The regions of uniqueness and non-uniqueness as derived in Section III and IV are indicated in Figure 11. As mentioned in paragraph $A$ of this section, the YLR region cannot be seen on the linear scale of this graph, a1though it exists for al1 $\mu$ for $\operatorname{sma} 11$ enough $\beta$. For negative $\mu$ there exists a region of uniqueness and non-uniqueness, so it is assumed that the two phases can coexist on some interface. This dashed interface line is drawn asymptoticaliy to the $\mu=a+2 b=3 b$ line derived 
by the perturbation method in Section $V$. As before, it is not known whether or not there is a critical temperature above which there can be but one phase. Other graphs with $a>2 b$ look quite similar even with slightly positivel a $(b<-17 a)$.

C. Posttive Patr Interaction. Assume that $a=3 b>0$. The region of uniqueness as derived in Section III is shown in Figure 12. As before, the YLR region boundary cannot be seen because of the scale. It is not known if there is a region where long range order is possible. In summary, all that is known about this case is that at high enough temperatures there exists but one phase (presumably, the random phase). 
VII. CONCLUSION.

It has been shown that the restricted dimer model with predominantly negative pair interactions (unless $a=2 b$, in which case the dimers have no preferential alignment) can show long-range order for suffictently low temperatures and high enough chemical potentlals. It is suggested that this region in the $\beta-\mu$ plane can be associated with an ordered close-packed state. It has been shown that there exists a region where there is short-range Independence (Dobrushin's Uniqueness Theorem). This region can be assoclated with the random phase. This region presumably has a strong connection with the high temperature analytic single phase region derived from the YLR Theorem. In any event, there is strong evidence that there is a region of long range order and a region of random order in the $\beta-\mu$ plane representing the two different phases. Hence, there must be a phase transition in passing from one region to the other. It has not been established whether or not there is a region of long range order for sufficiently positive pair interactions, although a random phase exists for high temperatures for all chemical potentials. 


\section{REFERENCES}

1. HEILMAN, 0.J., and E.H. LIEB: Phys. Rev. Letters 24, 1412 (1970).

2. GRUBER, C., and H. kUNZ: Commun. Math. Phys. 22, 133 (1971).

3. HEILMAN, 0.J.: Studies in Applied Mathematics 50, 385 (1971).

4. RUNNELS, L.K., and J.B. HUBBARD: J. Stat. Phys. 6, 1 (1972).

5. RUELIE, D.: Phys. Rev. Letters 26, 832 (1971).

6. DOBRUSHIN, R.L.: Functional Ana1. App1. 2, 292 (1968).

7. DOBRUSHIN, R.L.: Functional Ana1. App1. 2, 302 (1968).

8. HEILMAN, O.J.: Lettre al Nuovo Cimento 3, 95 (1972).

9. GRIFFITHS, R.: Phys. Rev. 136, 1437 (1964).

10. LEBOWITZ, J.L., and G. GALIAVOTTI: J. Math Phys. 12, 1129 (1971).

11. GALLAVOTTI, G., S. MIRACLE-SOLE, and D.W. ROBINSON: Phys. Letters 25 A, 493. (1971).

12. RUELIE, D.: Statistical Mechanics, 136 (1969).

13. PEIERLS, R.: Proc. Cambridge Phil. Soc. 32,477 (1936).

14. GRIFFITHS, R.: J. Math. Phys. 2, 1284 (1968).

15. RUNNELS, L.K., J.P. SALVANT, and H.R. STREIFFER: J. Chem Phys. 52, $2352(1970)$ 

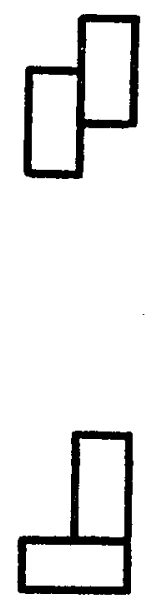

$\theta$

Figure 1 



Figure 2 


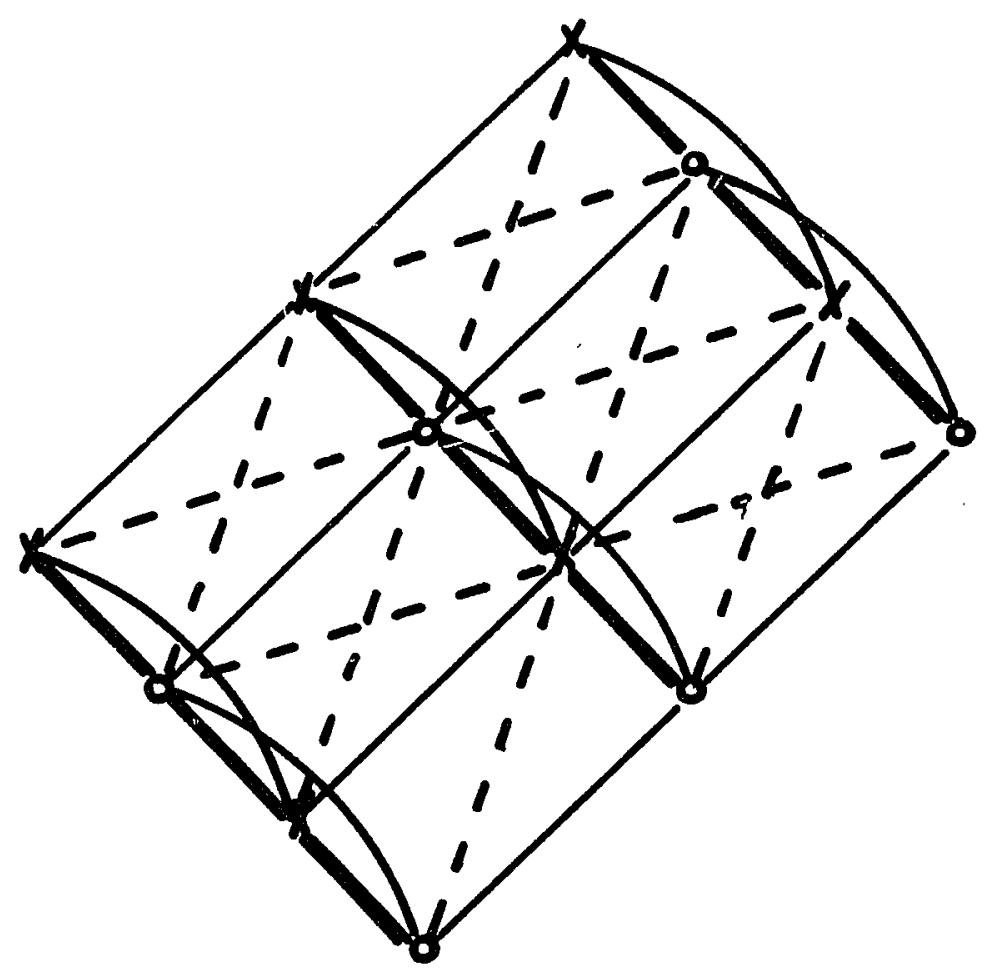

Figure 3 


$$
f_{0}
$$




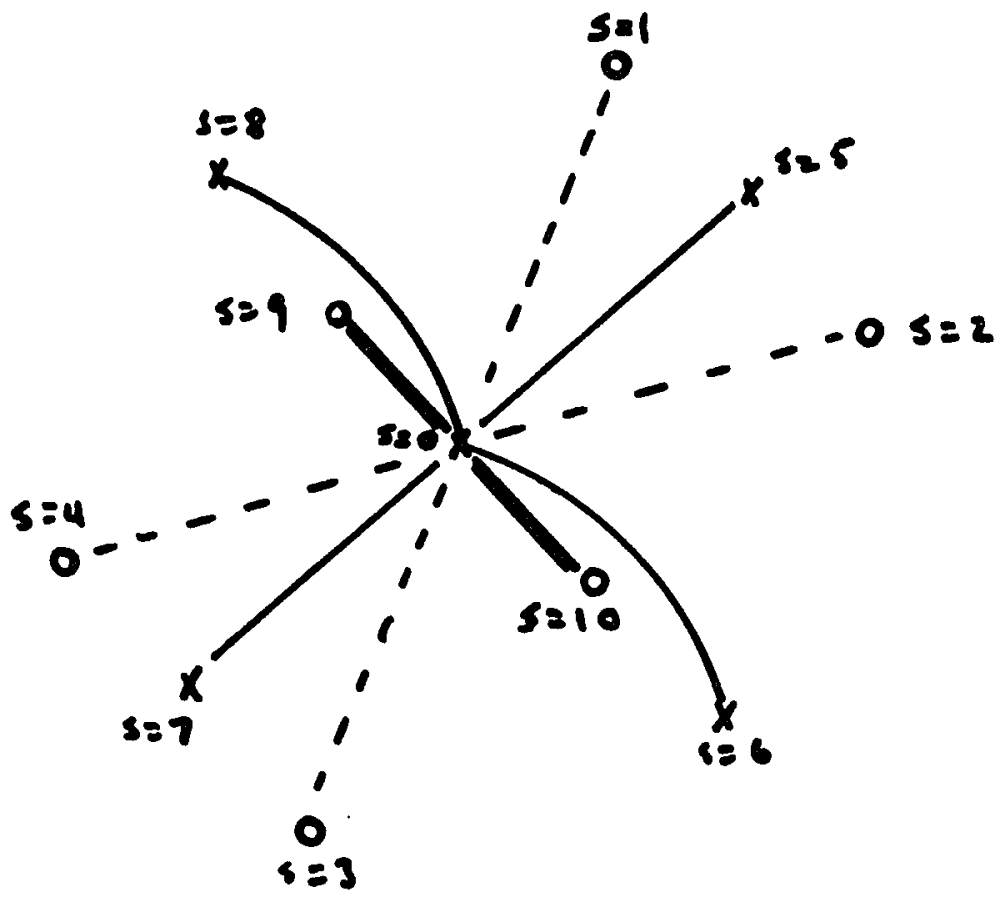

Figure 5 




Figure 6 


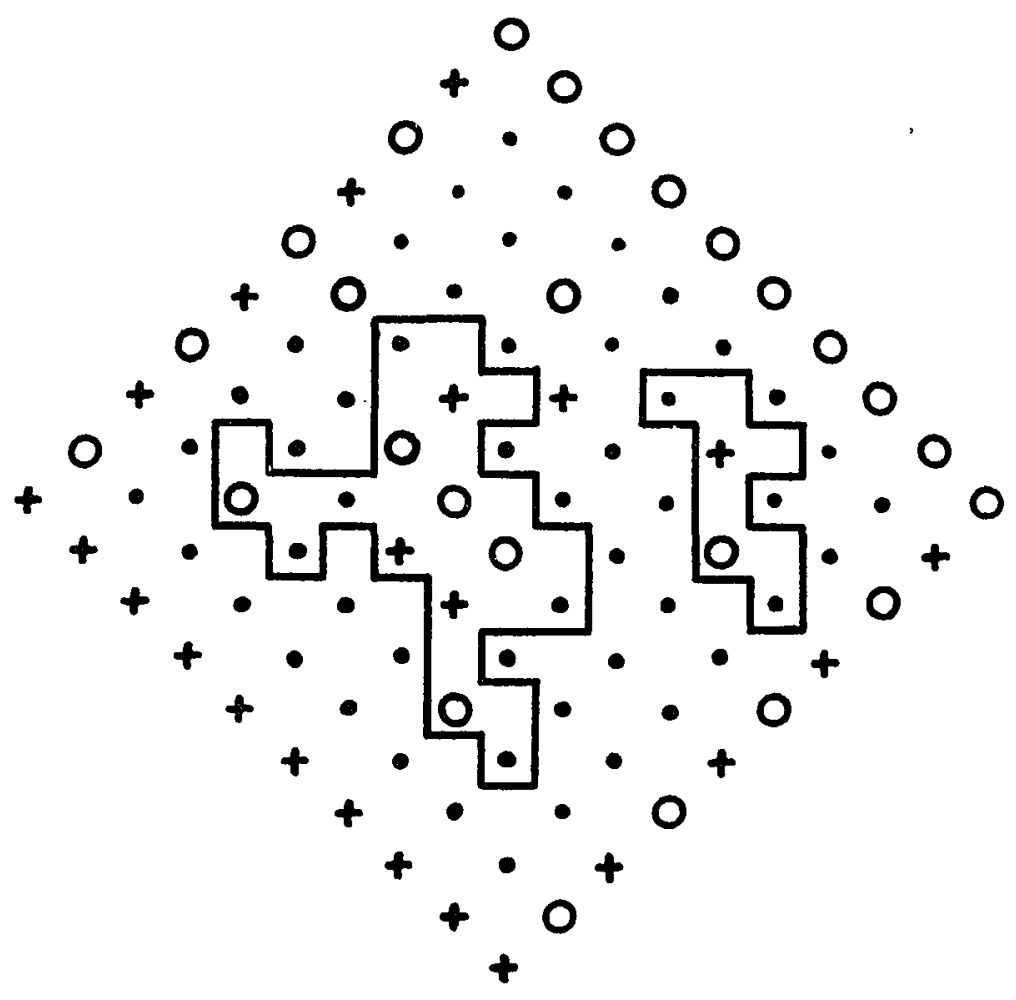

Figure 7 


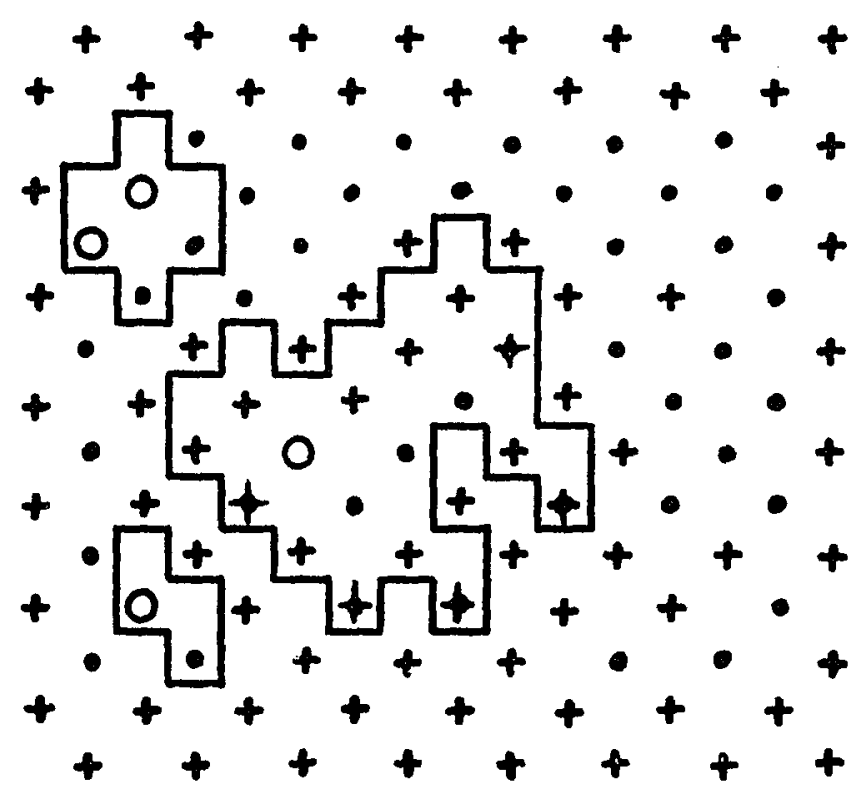

Figure 8 


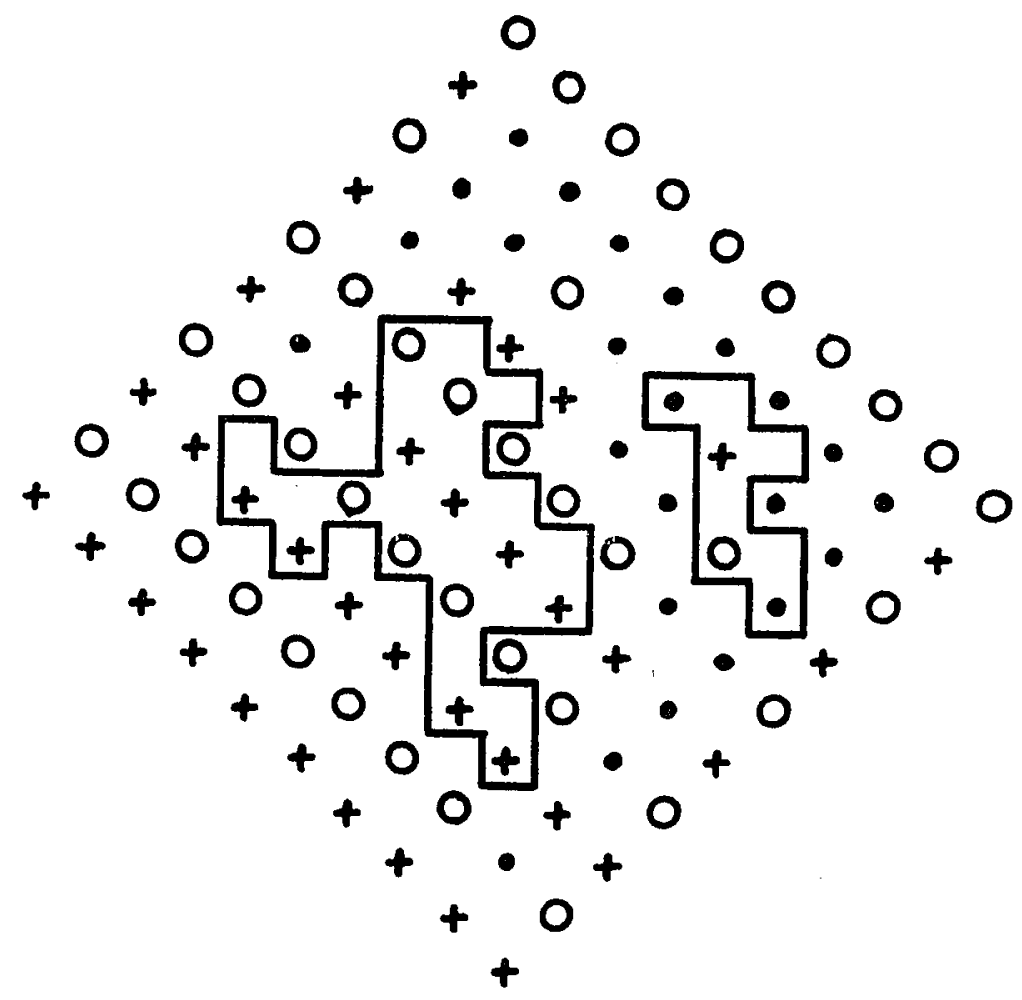

Figure 9 


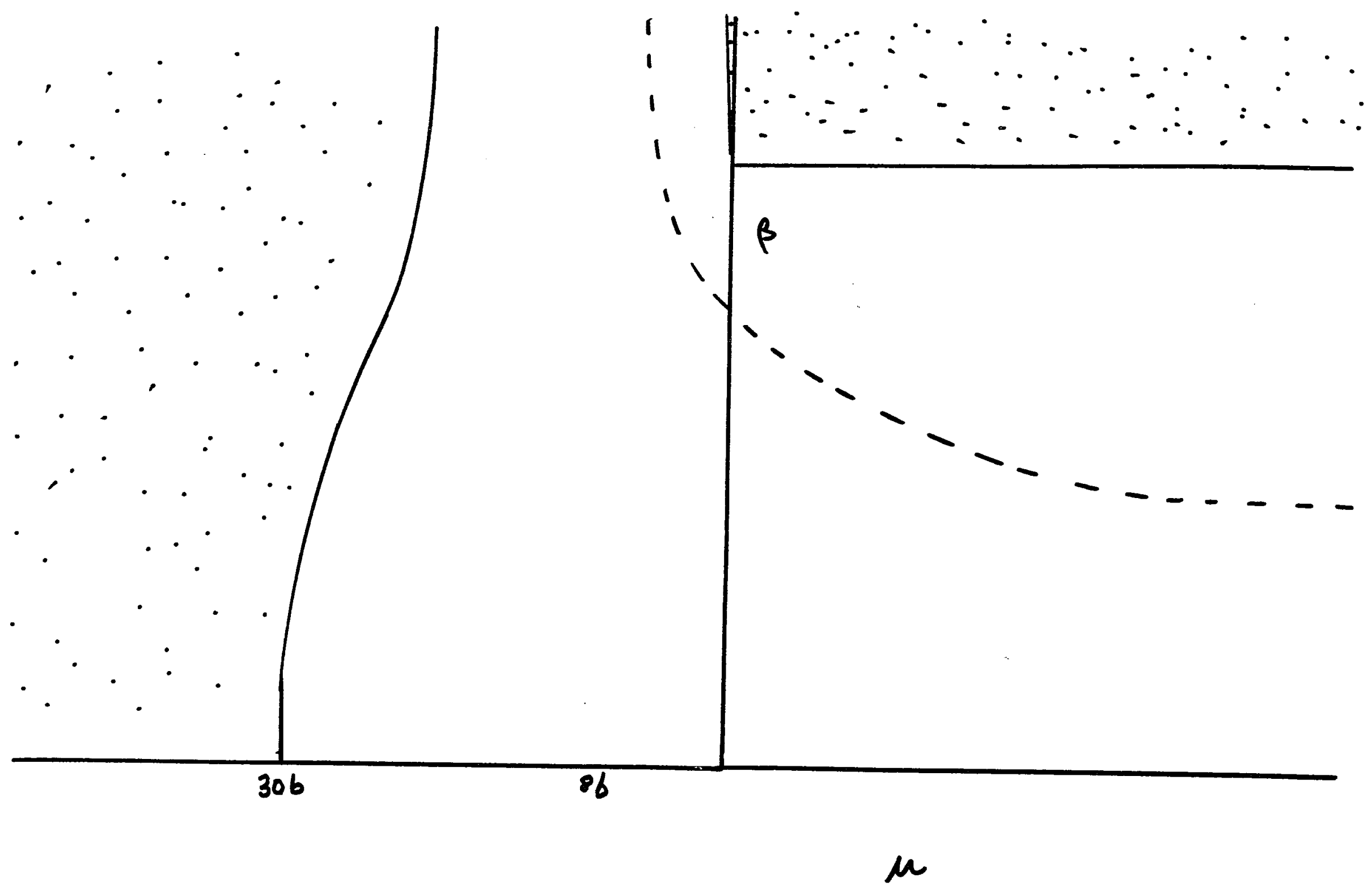

Figure 10 


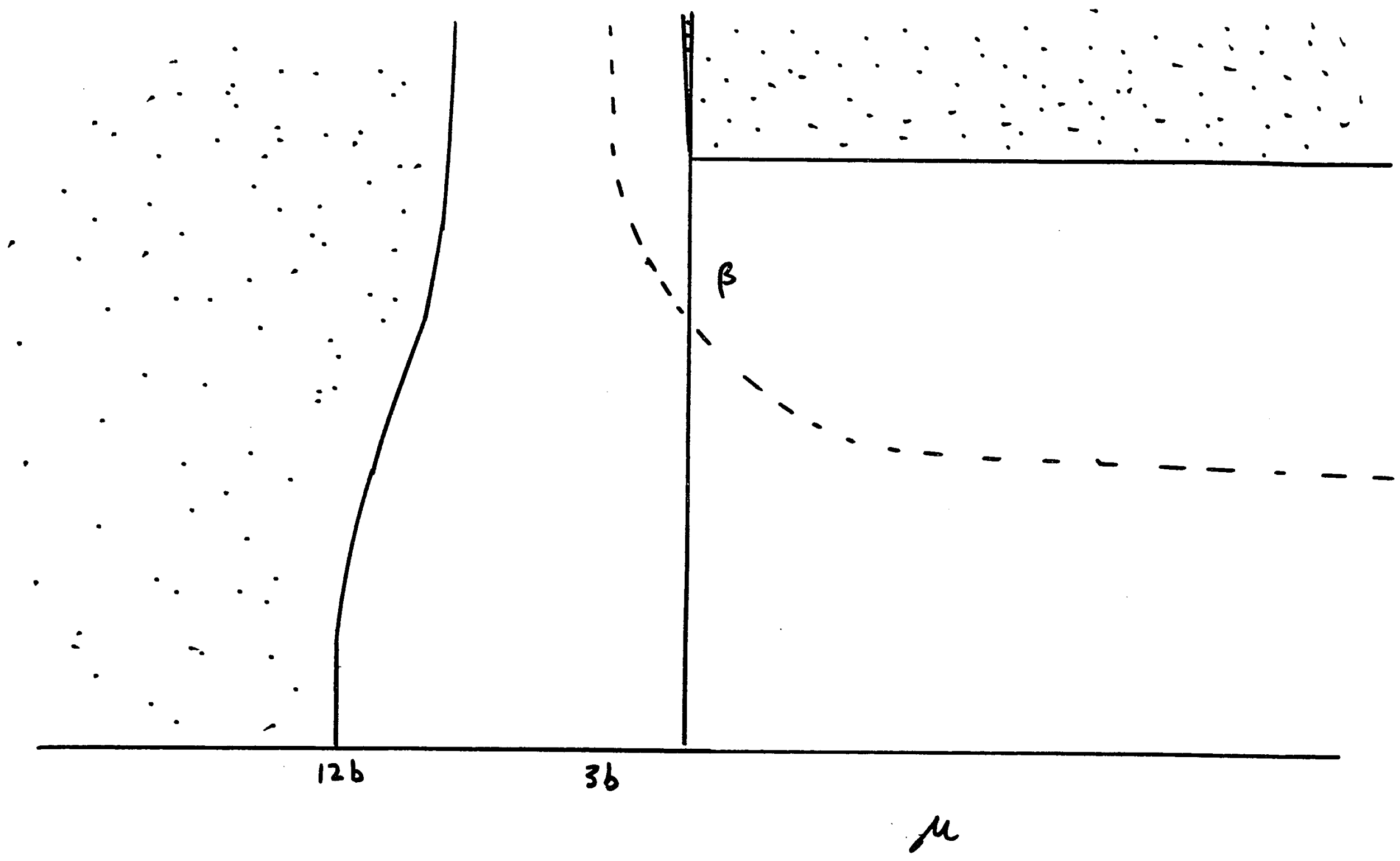

Figure 11 


$$
1
$$




\section{VITA}

Ben C. Freasier was born at Brawley, California, on February 16, 1945. He attended Ruston High School, and was graduated May 1963. He recelved his B.S. In Physics from Louistana State University in 1967. He entered the Graduate School of the Louisiana State University in September 1967, where he is presently a candidate for the Doctor of Philosophy Degree.

He is presently married to the former Linda Rowlands of Clarence, Louisiana. 
Candidate: Benjamin C. Freasier

Major Field: Chemical Physics

Title of Thesis: Equilibrium States of A Dimer Model with Angular Forces Approved:

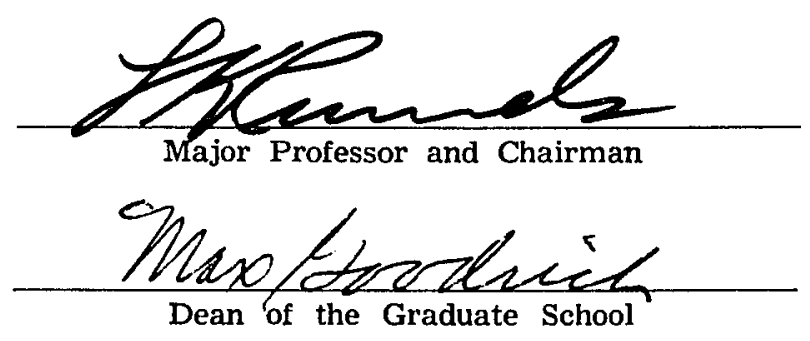

EXAMINING COMMITTEE:


Date of Examination:

March 1, 1973 